\title{
SOME CLASSES OF QUANTALE MORPHISMS
}

\section{GEORGE GEORGESCU}

Faculty of Mathematics and Computer Science

University of Bucharest

Bucharest

Romania

e-mail: georgescu.capreni@yahoo.com

\begin{abstract}
This paper concerns some types of coherent quantale morphisms: Baer, minimalisant, quasi rigid, quasi $r$-and quasi $r *$-quantale morphisms. Firstly, we study how the reticulation functor $L(\cdot)$ preserves the properties that define these types of quantale morphisms. Secondly, we prove some characterization theorems for quasi rigid, quasi $r$ - and quasi $r *$-quantale morphisms. These theorems extend some results existing in the literature of ring extensions and frame extensions.
\end{abstract}

2020 Mathematics Subject Classification: 06F07.

Keywords and phrases: coherent quantale, reticulation of a quantale, quantale morphisms, transfer properties.

Received March 5, 2021; Revised April 19, 2021

(C) 2021 Scientific Advances Publishers

This work is licensed under the Creative Commons Attribution International License (CC BY 3.0).

http://creativecommons.org/licenses/by/3.0/deed.en_US

Open Access (cc) (1)




\section{Introduction}

The papers ([5], [22]) studied some important classes of ring morphisms and ring extensions. Among them we mention Baer, minimalisant, flat ring morphisms and rigid, $r$ - and $r *$-ring extensions (resp., quasi rigid, quasi $r$-and quasi $r *$-ring extensions).

The quantales are multiplicative complete lattices that extend the lattices of ideals in (unital) commutative rings, as well as other lattices of congruences $[23,11,21]$. The reticulation of a quantale $A$ is a bounded distributive lattice $L(A)$ whose prime spectrum is homeomorphic to the $m$-prime spectrum of $A$ (cf. $[13,8]$ ). In fact, the reticulation construction provides a covariant functor $L(\cdot)$ from the category of coherent quantales to the category of bounded distributive lattices (see [8]).

This paper concerns some types of coherent quantale morphisms: Baer, minimalisant, quasi rigid, quasi $r$ - and quasi $r *$-quantale morphisms. These notions are abstractions of some remarkable types of morphisms and extensions studied in ring theory and frame theory (see $[3-6,22])$. Firstly, we study how the reticulation functor $L(\cdot)$ preserves the properties that define these types of quantale morphisms. Secondly, we prove some characterization theorems for quasi rigid, quasi $r$ - and quasi $r *$-quantale morphisms. These theorems extend some algebraic and topological results proved in [5] for ring extensions and in [3, 4] for frame extensions.

Now we shall present the structure of paper. Section 2 contains definitions and basic properties on quantales: arithmetical properties, radical and $m$-prime elements, Zariski and flat topologies on the $m$-prime spectrum (cf. $[23,11,14]$ ). Section 3 concerns some transfer properties of the reticulation regarding the $m$-prime elements and the annihilators. Section 4 deals with some elementary functorial properties of reticulation with emphasis on preservation of the annihilators. In Section 5, we find 
various descriptions of Baer and $w$-Baer quantale morphisms and we prove two preservation results related to these classes of quantale morphisms. In Section 6, we begin the study of $m$-quantale morphisms. Sections 7 and 8 concern the quasi $r$-and quasi $r *$-quantale morphisms respectively. We obtain algebraic and topological characterizations of these classes of quantale morphisms and some transfer results. Quasi rigid quantale morphisms are studied in Section 9.

\section{Preliminaries on Quantales}

This section contains some basic notions and results in quantale theory $([23,11])$. Let $(A, \bigvee, \wedge, \cdot, 0,1)$ be a quantale and $K(A)$ be the set of its compact elements. $A$ is said to be integral if $(A, \cdot, 1)$ is a monoid and commutative, if the multiplication - is commutative. A frame is a quantale in which the multiplication coincides with the meet [17]. The quantale $A$ is algebraic if any $a \in A$ has the form $a=\bigvee X$ for some subset $X$ of $K(A)$. An algebraic quantale $A$ is coherent if $1 \in K(A)$ and $K(A)$ is closed under the multiplication. Throughout this paper, the quantales are assumed to be integral and commutative. Often we shall write $a b$ instead of $a \cdot b$. We fix a quantale $A$.

Lemma 2.1 ([7]). For all elements $a, b, c$ of the quantale $A$ the following hold:

(1) If $a \vee b=1$, then $a \cdot b=a \wedge b$.

(2) If $a \vee b=1$, then $a^{n} \vee b^{n}=1$ for all integer numbers $n \geq 1$.

(3) If $a \vee b=a \vee c=1$, then $a \vee(b \cdot c)=a \vee(b \wedge c)=1$ 
On each quantale $A$ one can consider a residuation operation $a \rightarrow b=\bigvee\{x \mid a x \leq b\}$ and a negation operation $a^{\perp}=a^{\perp_{A}}$, defined by $a^{\perp}=a \rightarrow 0=\bigvee\{x \in A \mid a x=0\}$. Thus for all $a, b, c \in A$ the following equivalence holds: $a \leq b \rightarrow c$ if and only if $a b \leq c$, so $(A, \vee, \wedge, \cdot \rightarrow, 0,1)$ becomes a (commutative) residuated lattice.

In this paper, we shall use without mention the basic arithmetical properties of a residuated lattice [12]. An element $p<1$ of $A$ is m-prime if for all $a, b \in A, a b \leq p$ implies $a \leq p$ or $b \leq p$. If $A$ is an algebraic quantale, then $p<1$ is $m$-prime if and only if for all $c, d \in K(A), c d \leq p$ implies $c \leq p$ or $d \leq p$. Let us introduce the following notations: $\operatorname{Spec}(A)$ is the set of $m$-prime elements and $\operatorname{Max}(A)$ is the set of maximal elements of $A$. If $1 \in K(A)$, then for any $a<1$ there exists $m \in \operatorname{Max}(A)$ such that $a \leq m$. The same hypothesis $1 \in K(A)$ implies that $\operatorname{Max}(A) \subseteq \operatorname{Spec}(A)$.

The main example of quantale is the set $I d(R)$ of ideals of a (unital) commutative ring $R$ and the main example of frame is the set $\operatorname{Id}(L)$ of ideals of a bounded distributive lattice $L$. Thus the set $\operatorname{Spec}(R)$ of prime ideals in $R$ is the prime spectrum of the quantale $\operatorname{Id}(R)$ and the set of prime ideals in $L$ is the prime spectrum of the frame $\operatorname{Id}(L)$.

Following [23], the radical $\rho(a)=\rho_{A}(a)$ of an element $a \in A$ is defined by $\rho_{A}(a)=\Lambda\{p \in \operatorname{Spec}(A) \mid a \leq p\}$; if $a=\rho(a)$ then $a$ is a radical element. We shall denote by $R(A)$ the set of radical elements of $A$. The quantale $A$ is said to be semiprime if $\rho(0)=0$. 
Lemma 2.2 ([23]). For all elements $a, b \in A$ the following hold:

(1) $a \leq \rho(a)$;

(2) $\rho(a \wedge b)=\rho(a b)=\rho(a) \wedge \rho(b)$;

(3) $\rho(a)=1$ iff $a=1$;

(4) $\rho(a \vee b)=\rho(\rho(a) \vee \rho(b))$;

(5) $\rho(\rho(a))=\rho(a)$;

(6) $\rho(a) \vee \rho(b)=1$ iff $a \vee b=1$;

(7) $p\left(a^{n}\right)=\rho(a)$, for all integer $n \geq 1$.

Lemma 2.3 ([19]). Let $A$ be a coherent quantale and $a \in A$. Then

(1) $\rho(a)=\bigvee\left\{c \in K(A) \mid c^{k} \leq a\right.$ for some integer $\left.k \geq 1\right\}$;

(2) For any $c \in K(A), c \leq \rho(a)$ iff $c^{k} \leq a$ for some $k \geq 1$;

(3) $A$ is semiprime if and only if for any integer $k \geq 1, c^{k}=0$ implies $c=0$.

Let $A$ be a quantale such that $1 \in K(A)$. For any $a \in A$, denote $D_{A}(a)=D(a)=\{p \in \operatorname{Spec}(A) \mid a \leftleftarrows p\} \quad$ and $\quad V_{A}(a)=V(a)=\{p \in$ Spec $(A) \mid a \leq p\}$. Then $\operatorname{Spec}(A)$ is endowed with a topology whose closed sets are $(V(a))_{a \in A}$. If the quantale $A$ is algebraic, then the family $(D(c))_{c \in K(A)}$ is a basis of open sets for this topology. The topology introduced here generalizes the Zariski topology (defined on the prime spectrum $\operatorname{Spec}(R)$ of a commutative ring $R$ [1]) and the Stone topology (defined on the prime spectrum $\operatorname{Spec}_{I d}(L)$ of a bounded distributive lattice $L$ [2]). Then this topology will be also called the Zariski topology of $\operatorname{Spec}(A)$ and the corresponding topological space will be denoted by $\operatorname{Spec}_{Z}(A)$. According to [14], $\operatorname{Spec}_{Z}(A)$ is a spectral space in the sense of 
[15]. The flat topology on $\operatorname{Spec}(A)$ has as basis the family of the complements of compact open subsets of $\operatorname{Spec}_{Z}(A)$ (cf. [9, 17]). Recall from [14] that the family $\{V(c) \mid c \in K(A)\}$ is a basis of open sets for the flat topology on $\operatorname{Spec}(A)$. We shall denote by $\operatorname{Spec}_{F}(A)$ this topological space.

Let $L$ be a bounded distributive lattice. For any $x \in L$, denote $D_{I d}(x)=\left\{P \in \operatorname{Spec}_{I d}(L) \mid x \notin P\right\}$ and $V_{I d}(x)=\left\{P \in \operatorname{Spec}_{I d, Z}(L) \mid x \in P\right\}$.

The family $\left(D_{I d}(x)\right)_{x \in L}$ is a basis of open sets for the Stone topology on $\operatorname{Spec}_{I d}(L)$; this topological space will be denoted by $\operatorname{Spec}_{I d, Z}(L)$. We will denote by $\operatorname{Spec}_{I d, F}(L)$ the prime spectrum $\operatorname{Spec}_{I d}(L)$ endowed with the flat topology; the family $\left(V_{I d}(x)\right)_{x \in L}$ is a basis of open sets for the at topology.

An element $e$ of the quantale $A$ is a complemented element if there exists $f \in A$ such that $e \vee f=1$ and $e \wedge f=0$. The Boolean center of the quantale $A$ is the set $B(A)$ of complemented elements of $A$ (cf. [7, 16]).

The following lemma collects some elementary properties of the elements of $B(A)$.

Lemma 2.4 ([7, 16]). Let $A$ be a quantale and $a, b \in A, e \in B(A)$. Then the following properties hold:

(1) $a \in B(A)$ iff $a \vee a^{\perp}=1$;

(2) $a \wedge e=a e$

(3) $e \rightarrow a=e^{\perp} \vee a$;

(4) If $a \vee b=1$ and $a b=0$, then $a, b \in B(A)$;

(5) $(a \wedge b) \vee e=(a \vee e) \wedge(b \wedge e)$. 
Proposition 2.5. If $a \in A$, then $a^{\perp}=\wedge\left(V\left(a^{\perp}\right) \bigcap \operatorname{Min}(A)\right)$.

\section{Reticulation of a Coherent Quantale}

The reticulation $L(R)$ of a commutative ring $R$ was studied by many authors, but the main references on this topic remain [24, 17]. The reticulation $L(A)$ of a quantale $A$ (introduced in [13] as a generalization of the reticulation of a commutative ring) is a bounded distributive lattice whose prime spectrum $\operatorname{Spec}_{I d}(L(A))$ is homeomorphic to the prime spectrum $\operatorname{Spec}(A)$ of the quantale $A$. In this section, we shall recall from $[8,13]$ the axiomatic definition of the reticulation of the coherent quantale and some of its basic properties. Let $A$ be a coherent quantale and $K(A)$ the set of its compact elements.

Definition 3.1 ([8]). A reticulation of the quantale $A$ is a bounded distributive lattice $L$ together with a surjective function $\lambda: K(A) \rightarrow L$ such that for all $a, b \in K(A)$ the following properties hold:

(1) $\lambda(a \vee b) \leq \lambda(a) \vee \lambda(b)$;

(2) $\lambda(a b)=\lambda(a) \vee \lambda(b)$;

(3) $\lambda(a) \leq \lambda(b)$ iff $a^{n} \leq b$, for some integer $n \geq 1$.

In $([8,13])$, there were proven the existence and the uniqueness of the reticulation for each coherent quantale $A$; this unique reticulation will be denoted by $\left(L(A), \lambda_{A}: K(A) \rightarrow L(A)\right)$ or shortly $L(A)$.

We remark that the reticulation $L(R)$ of a commutative ring $R$ is isomorphic to the reticulation $L(\operatorname{Id}(R))$ of the quantale $\operatorname{Id}(R)$. 
Lemma 3.2 ([8]). For all elements $a, b \in K(A)$ the following properties hold:

(1) $a \leq b$ implies $\lambda_{A}(a) \leq \lambda_{A}(b)$;

(2) $\lambda_{A}(a \vee b)=\lambda_{A}(a) \vee \lambda_{A}(b)$;

(3) $\lambda_{A}(a)=1$ iff $a=1$;

(4) $\lambda_{A}(0)=0$;

(5) $\lambda_{A}(a)=0$ iff $a^{n}=0$, for some integer $n \geq 1$;

(6) $\lambda_{A}\left(a^{n}\right)=\lambda_{A}(a)$, for any integer $n \geq 1$;

(7) $\rho(a)=\rho(b)$ iff $\lambda_{A}(a)=\lambda_{A}(b)$;

(8) $\lambda_{A}(a)=0$ iff $a \leq \rho(0)$;

(9) If $A$ is semiprime, then $\lambda_{A}(a)=0$ implies $a=0$.

For any $a \in A$ and $I \in I d(L(A))$, let us denote $a^{*}=\left\{\lambda_{A}(c) \mid c \in K(A)\right.$, $c \leq a\}$ and $I_{*}=\bigvee\left\{c \in K(A) \mid \lambda_{A}(c) \in I\right\}$.

Lemma 3.3 ([8]). The following assertions hold:

(1) If $a \in A$, then $a^{*}$ is an ideal of $L(A)$ and $a \leq\left(a^{*}\right)_{*}$;

(2) If $I \in I d(L(A))$, then $\left(I_{*}\right)^{*}=I$;

(3) If $p \in \operatorname{Spec}(A)$, then $\left(p^{*}\right)_{*}=p$ and $p^{*} \in \operatorname{Spec}_{I d}(L(A))$;

(4) If $P \in \operatorname{Spec}_{I d}(L(A))$, then $P_{*} \in \operatorname{Spec}(A)$;

(5) If $c \in K(A)$, then $c^{*}=\left(\lambda_{A}(c)\right]$;

(6) If $c \in K(A)$ and $I \in I d(L(A))$, then $c \leq I_{*}$ iff $\lambda_{A}(c) \in I$; 
(7) If $a \in A$ and $I \in \operatorname{Id}(L(A))$, then $\rho(a)=\left(a^{*}\right)_{*}, a^{*}=(\rho(a))^{*}$ and $\rho\left(I_{*}\right)=I_{*} ;$

(8) If $c \in K(A)$ and $p \in \operatorname{Spec}(A)$, then $c \leq p$ iff $\lambda_{A}(c) \in p^{*}$.

Often the two previous lemmas shall be used in the proofs without mention.

According to Lemma 3.3, one can consider the following orderpreserving functions: $\delta_{A}: \operatorname{Spec}(A) \rightarrow \operatorname{Spec}_{I d}(L(A))$ and $\epsilon_{A}: \operatorname{Spec}_{I d}(L(A))$ $\rightarrow \operatorname{Spec}(A)$, defined by $\delta_{A}(p)=p^{*}$ and $\epsilon_{A}(P)=P_{*}$, for all $p \in \operatorname{Spec}(A)$ and $P \in \operatorname{Spec}_{I d}(L(A))$.

Lemma 3.4 ([8, 14]). The functions $\delta_{A}$ and $\epsilon_{A}$ are homeomorphisms w.r.t. the Zariski and the flat topologies, inverse to one another.

We also observe that $\delta_{A}$ and $\epsilon_{A}$ are also order-isomorphisms.

Lemma 3.5. Let $A$ be a semiprime coherent quantale and $a, b \in A$. Then $a^{\perp} \leq b^{\perp}$, if and only if $(a b)^{\perp}=b^{\perp}$.

Proof. Since $(a b)^{\perp} \geq b^{\perp}$ it suffices to prove that $a^{\perp} \leq b^{\perp}$, if and only if $(a b)^{\perp} \leq b^{\perp}$. If $(a b)^{\perp} \leq b^{\perp}$, then $a^{\perp} \leq(a b)^{\perp} \leq b^{\perp}$. Conversely, assume that $a^{\perp} \leq b^{\perp}$, therefore $a b c=0$. Thus $c b \leq a^{\perp} \leq b^{\perp}$, so $c b b=0$, hence we get $\lambda_{A}(b c)=\lambda_{A}(b) \wedge \lambda_{A}(c)=\lambda_{A}(c b b)=0$. Since $A$ is semiprime it follows that $b c=0$. Thus $b(a b)^{\perp}=0$, so one gets $(a b)^{\perp} \leq b^{\perp}$.

For a bounded distributive lattice $L$ we shall denote by $B(L)$ the Boolean algebra of the complemented elements of $L$. It is well-known that $B(L)$ is isomorphic to the Boolean center $B(\operatorname{Id}(L))$ of the frame $\operatorname{Id}(L)$ (see $[7,17])$. 
Let us fix a coherent quantale $A$.

Proposition 3.6 ([8]). The function $\left.\lambda_{A}\right|_{B(A)}: B(A) \rightarrow B(L(A))$ is a Boolean isomorphism.

If $L$ is bounded distributive lattice and $I \in \operatorname{Id}(L)$, then the annihilator of $I$ is the ideal $A n n_{L}(I)=A n n(I)=\{x \in I \mid x \wedge y=0$, for all $y \in L\}$.

Lemma 3.7 ([14]). If $c \in K(A)$ and $p \in \operatorname{Spec}(A)$, then $\operatorname{Ann}\left(\lambda_{A}(c)\right) \subseteq p^{*}$, if and only if $c \rightarrow \rho(0) \leq p$.

The next two propositions concern the behaviour of reticulation w.r.t. the annihilators.

Proposition 3.8 ([14]). If $a$ is an element of a coherent quantale, then Ann $\left(a^{*}\right)=(a \rightarrow \rho(0))^{*} ;$ if $A$ is semiprime, then Ann $\left(a^{*}\right)=\left(a^{\perp}\right)^{*}$.

Proposition 3.9 ([14]). Assume that $A$ is a coherent quantale. If I is an ideal of $L(A)$, then $(A n n(I))_{*}=I_{*} \rightarrow \rho(0)$; if $A$ is semiprime, then $(\operatorname{Ann}(I))_{*}=\left(I_{*}\right)^{\perp}$.

If $A$ is a quantale then we denote by $\operatorname{Min}(A)$ the set of minimal m-prime elements of $A ; \operatorname{Min}(A)$ is called the minimal prime spectrum of $A$. If $1 \in K(A)$ then for any $p \in \operatorname{Spec}(A)$ there exists $q \in \operatorname{Min}(A)$ such that $q \leq p$.

Proposition 3.10 ([18]). If $A$ is semiprime coherent quantale and $p \in \operatorname{Spec}(A)$, then $p \in \operatorname{Min}(A)$, if and only if for all $c \in K(A), c \leq p$ implies $c^{\perp} \npreceq p$.

We denote by $\operatorname{Min}_{Z}(A)$ (resp., $\operatorname{Min}_{F}(A)$ ) the topological space obtained by restricting the topology of $\operatorname{Spec}_{Z}(A)$ (resp., $\left.\operatorname{Spec}_{F}(A)\right)$ to $\operatorname{Min}(A)$. Then $\operatorname{Min}_{Z}(A)$ is homeomorphic to the space $\operatorname{Min}_{I d}(L(A))$ of minimal prime ideals in $L(A)$ with the Stone topology and $\operatorname{Min}_{F}(A)$ is 
homeomorphic to the space $\operatorname{Min}_{I d, F}(L(A))$ of minimal prime ideals in $L(A)$ with the flat topology (cf. Lemma 3.4). By [14], $\operatorname{Min}_{Z}(A)$ is a zerodimensional Hausdorff space and $\operatorname{Min}_{F}(A)$ is a compact $T_{1}$ space.

\section{Quantale Morphisms}

Let $A, B$ be two quantales and $u: A \rightarrow B$ be a function that preserves the arbitrary joins (in this case we have $u(0)=0$ ). Let us consider the function $\tilde{u}: B \rightarrow A$ defined by $\tilde{u}(b)=\bigvee\{a \in A \mid u(a) \leq b\}$, for any $b \in B$. It is well-known that $\tilde{u}$ is the right adjoint of $u: u(a) \leq b$ iff $a \leq \tilde{u}(b)$, for all $a \in A$ and $b \in B$ (see, e.g., Lemma 3.1 of [25]).

Let $A, B$ be two quantales. A function $f: A \rightarrow B$ is a morphism of quantales if it preserves the arbitrary joins and the multiplication (in this case we have $u(0)=0) ; f$ is an integral morphism if $f(1)=1$. If $u(K(A)) \subseteq K(B)$, then we say that $u$ preserves the compacts. If $u$ is an integral quantale morphism that preserves the compacts then it is called a coherent quantale morphism. In a similar manner one defines the frame morphisms, integral frame morphisms, coherent frame morphism, etc. (cf. $[3,4])$.

Let $f: R_{1} \rightarrow R_{2}$ be a morphism of (unital) commutative rings. If $I$ is an ideal of $R_{1}$, then $I^{e}$ will denote the extension of $I$ to $R_{2}$, i.e., the ideal $R_{2} f(I)$ generated by $f(I)$ in $R_{2}$ (cf. [1], p.9). Then the function $f^{\bullet}: \operatorname{Id}\left(R_{1}\right) \rightarrow \operatorname{Id}\left(R_{2}\right)$, defined by $f^{\bullet}(I)=I^{e}$, for any $I \in \operatorname{Id}\left(R_{1}\right)$, is a coherent quantale morphism. 
Let $f: L_{1} \rightarrow L_{2}$ be a morphism of bounded distributive lattices. If $I$ is an ideal of $L_{1}$, then $f^{\bullet}(I)$ is the lattice ideal $(f(I)]$ generated by $f(I)$ in $L_{2}$. Then the function $f^{\bullet}: \operatorname{Id}\left(L_{1}\right) \rightarrow \operatorname{Id}\left(L_{2}\right)$, defined by $I \mapsto f^{\bullet}(I)$, for any $I \in \operatorname{Id}\left(L_{1}\right)$, is a coherent frame morphism.

Lemma 4.1. Let $u: A \rightarrow B$ be a quantale morphism. If $q \in \operatorname{Spec}(B)$, then $\tilde{u}(q) \in \operatorname{Spec}(A)$. The function $\left.\tilde{u}\right|_{\operatorname{Spec}(B)}: \operatorname{Spec}(B) \rightarrow \operatorname{Spec}(A)$ is continuous w.r.t. the Zariski and the flat topology.

Proof. Let $a, b$ be two elements of $A$ such that $a b \leq \tilde{u}(q)$. Thus $u(a) u(b)=u(a b) \leq q$ (because $\tilde{u}$ is the right adjoint of $u$ ), hence $u(a) \leq q$ or $u(b) \leq q$. By applying again the adjointness property we get $a \leq \tilde{u}(q)$ or $b \leq \tilde{u}(q)$, so $\tilde{u}(q) \in \operatorname{Spec}(A)$.

Let us assume that $c \in K(A)$. For any $p \in \operatorname{Spec}(A)$ we have $p \in(\tilde{u})^{-1}\left(V_{A}(c)\right)$ iff $c \leq \tilde{u}(p)$ iff $p \in V_{B}(u(c))$, hence $(\tilde{u})^{-1}\left(V_{A}(c)\right)=$ $V_{B}(u(c))$ and $(\tilde{u})^{-1}\left(D_{A}(c)\right)=D_{B}(u(c))$. Then the two functions $\left.\tilde{u}\right|_{\operatorname{Spec}(B)}$ $: \operatorname{Spec}_{Z}(B) \rightarrow \operatorname{Spec}_{Z}(A) \quad$ and $\left.\quad \tilde{u}\right|_{\operatorname{Spec}(B)}: \operatorname{Spec}_{F}(B) \rightarrow \operatorname{Spec}_{F}(A) \quad$ are continuous.

In the rest of the section we shall assume that $A, B$ are two coherent quantales.

Proposition 4.2 ([8]). Let $u: A \rightarrow B$ be a coherent quantale morphism. Then there exists a morphism of bounded distributive lattices $L(u): L(A) \rightarrow L(B)$ such that the following diagram is commutative:

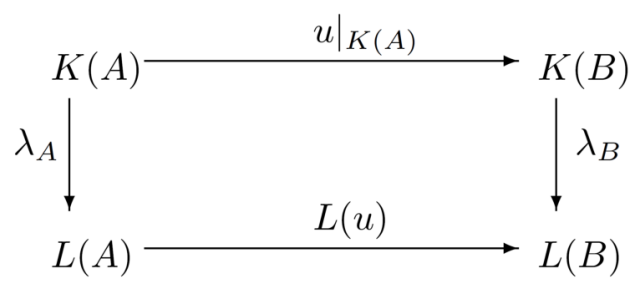


The following lemma is a well-known result in lattice theory.

Lemma 4.3. Let $f: L \rightarrow M$ be a morphism of bounded distributive lattices. For each $P \in \operatorname{Spec}_{I d}(L)$, there exists $Q \in \operatorname{Min}(M)$ such that $f^{-1}(Q) \subseteq P$.

Proof. For sake of completeness we shall present a short proof of this lemma. If $P \in \operatorname{Spec}_{I d}(L)$, then $1 \in f(L-P)$ and $f(L-P)$ is closed under meet. Then there exists $Q^{\prime} \in \operatorname{Spec}(M)$ such that $Q^{\prime} \bigcap f(L-P)=\emptyset$. Let $Q$ be a minimal prime ideal of $M$ such that $Q \subseteq Q^{\prime}$, so it is easy to see that $f^{-1}(Q) \subseteq P$.

Lemma 4.4. Let $u: A \rightarrow B$ be a coherent quantale morphism. If $p \in \operatorname{Spec}(A)$, then there exists $q \in \operatorname{Min}(B)$ such that $\tilde{u}(q) \leq p$.

Proof. Assume that $p \in \operatorname{Spec}(A)$, hence $p^{*} \in \operatorname{Spec}_{I d}(L(A))$. By Lemma 4.3, there exists $Q \in \operatorname{Min}_{I d}(L(B))$ such that $(L(u))^{-1}(Q) \subseteq p^{*}$. Thus $Q=q^{*}$ for some $q \in \operatorname{Min}(A)$, hence $(L(u))^{-1}\left(q^{*}\right) \subseteq p^{*}$.

We shall prove that $\tilde{u}(q) \leq p$. Let $c$ be a compact element of $A$ such that $c \leq \tilde{u}(q)$, so $u(c) \leq q$ (by the adjointness property). Therefore by using Proposition 4.2 we get $L(u)\left(\lambda_{A}(c)\right)=\lambda_{B}(u(c)) \in q^{*}$, hence $\lambda_{A}(c) \in(L(u))^{-1}\left(q^{*}\right) \subseteq p^{*}$. By using Lemma 3.3(8) one obtains $c \leq p$. We conclude that $\tilde{u}(q) \leq p$.

Corollary 4.5. Let $u: A \rightarrow B$ be a quantale morphism. If $p \in \operatorname{Min}(A)$, then there exists $q \in \operatorname{Min}(B)$ such that $\tilde{u}(q)=p$. 
Proposition 4.6. Let $u: A \rightarrow B$ be a coherent quantale morphism and assume that the quantales $A, B$ are semiprime.

(1) If $a \in A$, then $(u(a))^{\perp} B=\left(\left(L(u)\left(a^{*}\right)\right)_{*}\right)^{\perp_{B}}$;

(2) If $I \in I d(L(A))$, then $A n n_{L(B)}(L(u)(I))=A n n_{L(B)}\left(\left(u\left(I_{*}\right)\right)^{*}\right)$.

Proof. (1) For all $a \in A$ and $d \in K(B)$ we shall prove the following equivalence:

$$
d u(a)=0 \text { if and only if } d\left(\left(L(u)\left(a^{*}\right)\right)_{*}\right)=0 .
$$

Assume that $d u(a)=0$. Let $e$ be a compact element of $B$ such that $\lambda_{B}(e) \in L(a)\left(a^{*}\right)$, so there exists $c \in K(A)$ such that $c \leq a$ and $\lambda_{B}(e)=$ $L(u)\left(\lambda_{A}(c)\right)=\lambda_{B}(u(c))$ (the last equality is due by Proposition 4.2). Thus $d u(c) \leq d u(a)=0, \quad$ so $\quad \lambda_{B}(d e)=\lambda_{B}(d) \wedge \lambda_{B}(e)=\lambda_{B}(d) \wedge \lambda_{B}(u(c))=$ $\lambda_{B}(d u(c))=0$. Since $B$ is semiprime, by Lemma 3.3(9) we have $d e=0$, so the equalities $d\left(\left(L(u)\left(a^{*}\right)\right)_{*}\right)=d\left(\bigvee\left\{e \in K(B) \mid \lambda_{B}(e) \in L(u)\left(a^{*}\right)\right\}\right)=$ $\bigvee\left\{d e \mid e \in K(B), \lambda_{B}(e) \in L(u)\left(a^{*}\right)\right\}=0$ hold.

Conversely, let us suppose that $d\left(\left(L(u)\left(a^{*}\right)\right)_{*}\right)=0$. Let $c$ be a compact element of $A$ such that $c \leq a$, so $u(c) \in K(B)$ and $\lambda_{A}(c) \in a^{*}$, therefore we get $\lambda_{B}(u(c))=L(u)\left(\lambda_{A}(c)\right) \in L(u)\left(a^{*}\right)$. It follows that $u(c) \leq\left(L(u)\left(a^{*}\right)\right)_{*}$, hence $d u(c)=0$. Recall that the map $u$-preserves the arbitrary joins. Then the following equalities hold:

$$
d u(a)=d u(\bigvee\{c \in K(A) \mid c \leq a\})=\bigvee\{d u(c) \mid c \in K(A), c \leq a\}=0
$$


We have proven the equivalence (4.1), therefore the equality $(u(a))^{\perp_{B}}=$ $\left(\left(L(u)\left(a^{*}\right)\right)_{*}\right)^{\perp_{B}}$ holds.

(2) We shall prove that for any $d \in K(B)$ the following equivalence holds:

$$
\lambda_{B}(d) \in A n n_{L(B)}(L(u)(I)) \text { if and only if } \lambda_{B}(d) \in A n n_{L(B)}\left(\left(u\left(I_{*}\right)\right)^{*}\right) .
$$

Assume that $\lambda_{B}(d) \in A n n_{L(B)}(L(u)(I))$ and $y \in\left(u\left(I_{*}\right)\right)^{*}$, so there exists $e \in K(B)$ such that $e \leq u\left(I_{*}\right)$ and $y=\lambda_{B}(e)$. We remark that

$$
u\left(I_{*}\right)=u\left(\bigvee\left\{c \in K(A) \mid \lambda_{A}(c) \in I\right\}\right)=\bigvee\left\{u(c) \mid c \in K(A), \lambda_{A}(c) \in I\right\},
$$

therefore from $e \leq u\left(I_{*}\right)$ and $e \in K(B)$ we get $e \leq u(c)$, for some $c \in K(A)$ such that $\lambda_{A}(c) \in I$. Then $L(u)\left(\lambda_{A}(c)\right) \in L(u)(I)$, hence one gets $\lambda_{B}(d u(c))=\lambda_{B}(d) \wedge \lambda_{B}(u(c))=\lambda_{B}(d) \wedge L(u)\left(\lambda_{A}(c)\right)=0$. Since the quantale $B$ is semiprime we have $d u(c)=0$, so $d e=0$. Thus $\lambda_{B}(d) \wedge y$ $=\lambda_{B}(d) \wedge \lambda_{B}(e)=\lambda_{B}(d e)=0$, so $\lambda_{B}(d) \in A n n_{L(B)}\left(\left(u\left(I_{*}\right)\right)^{*}\right)$.

Conversely, assume that $\lambda_{B}(d) \in A n n_{L(B)}\left(\left(u\left(I_{*}\right)\right)^{*}\right)$. For any $z \in L(u)(I)$, there exists $c \in K(A)$ such that $\lambda_{A}(c) \in K(A)$ and $z=L(u)\left(\lambda_{A}(c)\right)=\lambda_{B}(u(c))$. Thus $\lambda_{B}(u(c)) \in L(u)(I)$, hence by using (4.3) we get $u(c) \leq u\left(I_{*}\right)$, therefore $z=\lambda_{B}(u(c)) \in\left(u\left(I_{*}\right)\right)^{*}$. By applying the hypothesis, we get $\lambda_{B}(d) \wedge z=0$, hence $\lambda_{B}(d) \in A n n_{L(B)}(L(u)(I))$. 


\section{Baer Quantale Morphisms}

Let $f: R \rightarrow Q$ be a morphism of commutative rings. Following [22], we say that $f$ is a Baer ring morphism if for all $I, j \in I d(R), A n n_{R}(I)=$ $A n n_{R}(J)$ implies $A n n_{Q}\left(I^{e}\right)=A n n_{Q}\left(J^{e}\right) ; f$ is said to be a $w$-Baer ring morphism if for all $x, y \in R, A n n_{R}(x)=A n n_{R}(y)$ implies $A n n_{Q}(f(x))=$ $A n n_{Q}(f(y))$.

Let $f: L \rightarrow M$ be a morphism of bounded distributive lattices. We say that $f$ is a Stone lattice morphism if for all $I, J \in I d(L), A n n_{L}(I)=$ $A n n_{L}(J)$ implies $A n n_{M}\left(I^{e}\right)=A n n_{M}\left(J^{e}\right) ; f$ is said to be a $w$-Stone lattice morphism if for all $x, y \in M, A n n_{L}(x)=A n n_{L}(y)$ implies $A n n_{M}(f(x))=A n n_{M}(f(y))$.

The previous definitions will be extended from ring and lattice morphisms to quantale morphisms. Let $u: A \rightarrow B$ be a quantale morphism. Then $u$ is said to be a Baer quantale morphism if for all $a, b \in A, a^{\perp_{A}}=b^{\perp_{A}}$ implies $(u(a))^{\perp_{B}}=(u(b))^{\perp_{B}} ; u$ is said to be a $w$-Baer quantale morphism if for all $a, b \in K(A), a^{\perp_{A}}=b^{\perp_{A}}$ implies $(u(a))^{\perp B}=(u(b))^{\perp} B$. The notions of Baer and $w$-Baer frame morphisms are defined in a similar manner.

A morphism $f: R \rightarrow Q$ of commutative rings is a Baer (resp., $w$-Baer) ring morphism if and only if $f^{\bullet}: \operatorname{Id}(R) \rightarrow \operatorname{Id}(Q)$ is a Baer (resp., $w$-Baer) quantale morphism. A morphism $f: L \rightarrow M$ of bounded distributive lattices is a Stone (resp., $w$-Stone) lattice morphism if and only if $f^{\bullet}: \operatorname{Id}(L) \rightarrow \operatorname{Id}(M)$ is a Baer (resp., $w$-Baer) frame morphism.

The class of Baer (resp., $w$-Baer) quantale morphisms is closed under the composition. The following result generalizes the main part of Proposition 8 of [22]. 
Proposition 5.1. Let us consider two semiprime coherent quantales $A, B$ and a coherent quantale morphism $u: A \rightarrow B$. Then the following assertions are equivalent:

(1) u is a Baer quantale morphism;

(2) For any $a \in A, a^{\perp_{A}}=0$ implies $(u(a))^{\perp_{B}}=0$;

(3) For all $a, b \in A, a^{\perp_{A}} \leq b^{\perp_{A}}$ implies $(u(a))^{\perp_{B}} \leq(u(b))^{\perp_{B}}$;

(4) For any $a \in A,\left(u\left(a^{\perp_{A}}\right)\right)^{\perp_{B} \perp_{B}}=(u(a))^{\perp_{B}}$;

(5) For any $b \in B,\left(\left.b^{\perp_{B}}\right|_{A}\right)^{\perp_{A}}=0$ implies $b=0$, whenever $\left.b^{\perp_{B}}\right|_{A}$ is the element of $A$ defined by $\left.b^{\perp B}\right|_{A}=\bigvee\{c \in A \mid u(c) b=0\}$.

Proof. We shall assume that $a$ and $b$ are arbitrary elements of the quantale $A$.

(1) $\Rightarrow(2)$ Assume that $u$ is a Baer quantale morphism. If $a^{\perp_{A}}=0$, then $a^{\perp A}=1^{\perp}$, hence $(u(a))^{\perp_{B}}=(u(1))^{\perp_{B}}=1^{\perp_{B}}=0$.

(2) $\Rightarrow$ (4) Since $\left(a \vee a^{\perp_{A}}\right)^{\perp_{A}}=a^{\perp_{A}} \wedge a^{\perp_{A} \perp_{A}}=0$, by applying the hypothesis (2) we get $\left(u\left(a \vee a^{\perp_{A}}\right)\right)^{\perp_{B}}=0$, hence $(u(a))^{\perp_{B}} \wedge\left(u\left(a^{\perp_{A}}\right)\right)^{\perp_{B}}=$ $\left(u(a) \vee u\left(a^{\perp_{A}}\right)\right)^{\perp_{B}}=\left(u\left(a \vee a^{\perp_{A}}\right)\right)^{\perp_{B}}=0$. Thus $(u(a))^{\perp_{B}}\left(u\left(a^{\perp_{A}}\right)\right)^{\perp_{B}}=0$, so we get the inequality $\left(u\left(a^{\perp_{A}}\right)\right)^{\perp_{B} \perp_{B}} \geq(u(a))^{\perp_{B}}$.

On the other hand, $u(a) u\left(a^{\perp_{A}}\right)=u\left(a a^{\perp_{A}}\right)=u(0)=0 \quad$ implies $u(a) \leq\left(u\left(a^{\perp_{A}}\right)\right)^{\perp_{B}}$, therefore the converse inequality $\left(u\left(a^{\perp_{A}}\right)\right)^{\perp_{B} \perp_{B}} \leq$ $(u(a))^{\perp B}$ holds. Thus $u$ is a Baer quantale morphism. 
(4) $\Rightarrow$ (1) If $a^{\perp_{A}}=b^{\perp_{A}}$, then

$$
(u(a))^{\perp_{B}}=\left(u\left(a^{\perp_{A}}\right)\right)^{\perp_{B} \perp_{B}}=\left(u\left(b^{\perp_{A}}\right)\right)^{\perp_{B} \perp_{B}}=(u(b))^{\perp_{B}} .
$$

(3) $\Rightarrow$ (1) Obviously.

(1) $\Rightarrow$ (3) By using Lemma 3.5, the following implications hold:

$$
a^{\perp_{A}} \leq b^{\perp_{A}} \Rightarrow(a b)^{\perp_{A}}=b^{\perp_{A}}(u(a b))^{\perp_{B}}=(u(b))^{\perp_{B}} .
$$

Observing that $u(a b)=u(a) u(b) \leq u(a)$ implies $(u(a))^{\perp B} \leq(u(a b))^{\perp_{B}}$ it follows that $a^{\perp_{A}} \leq b^{\perp_{A}}$ implies $(u(a))^{\perp_{B}} \leq(u(b))^{\perp_{B}}$.

(2) $\Rightarrow$ (5) Assume that $\left(\left.b^{\perp_{B}}\right|_{A}\right)^{\perp_{A}}=0$ so $\left(u\left(\left.b^{\perp_{B}}\right|_{A}\right)\right)^{\perp_{B}}=0$ (by applying the hypothesis (2)). We remind that $u$ preserves arbitrary joins, hence the following equalities hold: $b u\left(\left.b^{\perp_{B}}\right|_{A}\right)=b u(\bigvee\{c \in A \mid u(c) b=0\}=$ $b \bigvee\{u(c) \mid u(c) b=0\}=\bigvee\{b u(c) \mid c \in A, u(c) b=0\}=0$. Therefore $b \leq\left(u\left(b^{\perp} B \mid\right.\right.$ A) $)^{\perp_{B}}=0$, so $b=0$.

(5) $\Rightarrow$ (2) Assume that $a \in A$ and $a^{\perp A}=0$. For any $c \in B$, the following implications hold:

$$
\begin{aligned}
c & \leq(u(a))^{\perp_{B}} \Rightarrow c u(a)=0 \Rightarrow a \leq\left. c^{\perp_{B}}\right|_{A} \Rightarrow\left(\left.c^{\perp_{B}}\right|_{A}\right)^{\perp_{A}} \\
& \leq a^{\perp_{A}}=0 \Rightarrow c=0 .
\end{aligned}
$$

In particular, we obtain $(u(a))^{\perp_{B}}=0$.

For the rest of section, let us fix two semiprime coherent quantales $A, B$ and a coherent quantale morphism $u: A \rightarrow B$. We shall describe the way in which the reticulation functor $L(\cdot)$ transforms the Baer (resp., $w$-Baer) quantale morphisms into the Stone (resp., $w$-Stone) lattice morphisms. 
Theorem 5.2. The following assertions are equivalent:

(1) $u$ is a Baer quantale morphism;

(2) L(u) is a Stone lattice morphism.

Proof. $\quad(1) \Rightarrow(2)$ Let $I, J$ be two ideals of $L(A)$ such that $A n n_{L(A)}(I)=A n n_{L(A)}(J)$. Recall that the quantales $A$ and $B$ are semiprime. According to Proposition 3.9, $\left(I_{*}\right)^{\perp A}=\left(A n n_{L(A)}(I)\right)_{*}=$ $\left(A n n_{L(A)}(J)\right)_{*}=\left(J_{*}\right)^{\perp} A$, therefore, by applying the hypothesis that $u$ is a Baer quantale morphism, it follows that $\left(u\left(I_{*}\right)\right)^{\perp_{B}}=\left(u\left(J_{*}\right)\right)^{\perp_{B}}$. Then by using Proposition 3.8, we get

$$
A n n_{L(B)}\left(\left(u\left(I_{*}\right)\right)^{*}\right)=\left(\left(u\left(I_{*}\right)\right)^{\perp_{B}}\right)^{*}=\left(\left(u\left(J_{*}\right)\right)^{\perp_{B}}\right)^{*}=A n n_{L(B)}\left(\left(u\left(J_{*}\right)\right)^{*}\right) .
$$

By Proposition 4.6(2) we get $A n n_{L(B)}(L(u)(I))=A n n_{L(B)}(L(u)(J))$, so $L(u)$ is a Stone lattice morphism.

(2) $\Rightarrow$ (1) Let $a, b$ be two elements of $A$ such that $a^{\perp_{A}}=b^{\perp_{A}}$. By Proposition 3.8, we have $A n n_{L(A)}\left(a^{*}\right)=\left(a^{\perp A}\right)^{*}=\left(b^{\perp A}\right)^{*}=A n n_{L(A)}\left(b^{*}\right)$. Since $L(u)$ is a Stone lattice morphism we get $A n n_{L(B)}\left(L(u)\left(a^{*}\right)\right)=$ $A n n_{L(B)}\left(L(u)\left(b^{*}\right)\right)$, therefore $\left(A n n_{L(B)}\left(L(u)\left(a^{*}\right)\right)\right)_{*}=\left(A n n_{L(B)}\left(L(u)\left(b^{*}\right)\right)\right)_{*}$. In accordance with Proposition 3.9 we get $\left(\left(L(u)\left(a^{*}\right)\right)_{*}\right)^{\perp_{B}}=((L(u)$ $\left.\left.\left(b^{*}\right)\right)_{*}\right)^{\perp_{B}}$, hence $(u(a))^{\perp_{B}}=(u(b))^{\perp_{B}}$ (by using Proposition 4.6(1)). 
Lemma 5.3. For any $c \in K(A), c^{\perp_{A}}=(\rho(c))^{\perp_{A}}$.

Proof. Firstly we observe that $c \leq \rho(c)$ implies $c^{\perp_{A}} \geq(\rho(c))^{\perp_{A}}$. In order to show that $c^{\perp A} \leq(\rho(c))^{\perp A}$, let us consider a compact element $c$ of $A$ such that $d \leq c^{\perp} A$, hence $d c=0$. Let $c$ be a compact element of $A$ such that $e^{n} \leq c$ for some integer $n \geq 1$, therefore $d e^{n} \leq d c=0$. It follows that the following equalities hold: $d \rho(c)=d\left(\bigvee\left\{e \in K(A) \mid e^{n} \leq c\right.\right.$, for some integer $n \geq 1\})=\bigvee\left\{d e \in K(A) \mid e^{n} \leq c\right.$, for some integer $\left.n \geq 1\right\}=0$.

Thus $d \leq(\rho(c))^{\perp_{A}}$, so we conclude that $c^{\perp_{A}} \leq(\rho(c))^{\perp_{A}}$.

Theorem 5.4. The following assertions are equivalent:

(1) u is a w-Baer quantale morphism;

(2) $L(u)$ is a w-Stone lattice morphism.

Proof. (1) $\Rightarrow(2)$ Assume that $u$ is a $w$-Baer quantale morphism and $c, d$ are two compact elements of $A$ such that $A n n_{L(A)}\left(\lambda_{A}(c)\right)=$ $A n n_{L(A)}\left(\lambda_{A}(d)\right)$, so, by applying Proposition 3.9, the following equalities hold:

$\left(\left(\lambda_{A}(c)\right]\right)^{\perp_{A}}=\left(A n n_{L(A)}\left(\left(\lambda_{A}(c)\right]\right)\right)_{*}=\left(A n n_{L(A)}\left(\left(\lambda_{A}(d)\right]\right)\right)_{*}=\left(\left(\lambda_{A}(d)\right]\right)^{\perp} A$.

We remark that $\left(\lambda_{A}(c)\right]_{*}=\left(c^{*}\right)_{*}=\rho(c)$ and $\left(\lambda_{A}(d)\right]_{*}=\left(d^{*}\right)_{*}=\rho(d)$ (cf. Lemma 3.3(5) and (7)), hence, by using Lemma 5.4, we get $c^{\perp_{A}}=(\rho(c))^{\perp_{A}}=(\rho(d))^{\perp_{A}}=c^{\perp_{A}}$. Then $(u(c))^{\perp_{B}}=(u(d))^{\perp_{B}} \quad$ (by the hypothesis (1)), hence, by using Proposition 3.8, the following hold:

$$
A n n_{L(B)}\left((u(c))^{*}\right)=\left((u(c))^{\perp B}\right)^{*}=\left((u(d))^{\perp B}\right)^{*}=A n n_{L(B)}\left((u(d))^{*}\right) .
$$


By Proposition 4.2 and Lemma 3.3(5), we obtain the following equalities hold: $A n n_{L(B)}\left(L(u)\left(\lambda_{A}(c)\right)\right)=A n n_{L(B)}\left(\lambda_{B}(u(c))\right)=A n n_{L(B)}\left((u(c))^{*}\right)=$ $A n n_{L(B)}\left((u(d))^{*}\right)=\cdots=A n n_{L(B)}\left(L(u)\left(\lambda_{A}(d)\right)\right)$. We conclude that $L(u)$ is a $w$-Stone lattice morphism.

$(2) \Rightarrow(1)$ Assume that $L(u)$ is a $w$-Stone lattice morphism. Let $c, d$ be two compacts of $A$ such that $c^{\perp_{A}}=d^{\perp_{A}}$. By using Lemma 3.3(5), the following equalities hold: $A n n_{L(A)}\left(\lambda_{A}(c)\right)=A n n_{L(A)}\left(c^{*}\right)=\left(c^{\perp_{A}}\right)^{*}=$ $\left(d^{\perp A}\right)^{*}=A n n_{L(A)}\left(d^{*}\right)=A n n_{L(A)}\left(\lambda_{A}(d)\right)$. Applying the hypothesis and Proposition 4.2, we get $A n n_{L(B)}\left(\lambda_{B}(u(c))\right)=A n n_{L(B)}\left(L(u)\left(\lambda_{B}(c)\right)\right)=$ $A n n_{L(B)}\left(L(u)\left(\lambda_{B}(d)\right)\right)=A n n_{L(B)}\left(\lambda_{B}(u(d))\right)$.

In virtue of Proposition 3.8, we get $\left((u(c))^{\perp} B\right)^{*}=A n n_{L(B)}\left(\lambda_{B}(u(c))\right)=$ $A n n_{L(B)}\left(\lambda_{B}(u(d))\right)=\left((u(d))^{\perp_{B}}\right)^{*}$, therefore $\rho\left((u(c))^{\perp B}\right)=\left(\left((u(c))^{\perp_{B}}\right)^{*}\right)_{*}=$ $\left(\left((u(d))^{\perp_{B}}\right)^{*}\right)_{*}=\rho\left((u(d))^{\perp_{B}}\right)$. By using Lemma 5.3 we obtain $(u(c))^{\perp_{B}}=$ $(u(c))^{\perp_{B}}$, so $u$ is a $w$-Baer quantale morphism.

\section{Minimalisant Quantale Morphisms}

Let $f: R \rightarrow S$ be a morphism of commutative rings. By [22], $f$ is said to be a minimalisant ring morphism (= $m$-ring morphism) if for each $Q \in \operatorname{Min}(S), f^{-1}(Q) \in \operatorname{Min}(R)$. This notion can be generalized to the quantale framework: a quantale morphism $u: A \rightarrow B$ is said to be a minimalisant quantale morphism (= $m$-quantale morphism) if for each $q \in \operatorname{Min}(B), \tilde{u}(q) \in \operatorname{Min}(A)$. It is clear that a ring morphism $f: R \rightarrow S$ is an $m$-ring morphism if and only if $f^{\bullet}: \operatorname{Id}(R) \rightarrow \operatorname{Id}(S)$ is an $m$-quantale morphism. 
Let $f: L \rightarrow M$ be a morphism of bounded distributive lattices. Then $f$ is said to be a minimalisant lattice morphism (= $m$-lattice morphism) if for each $Q \in \operatorname{Min}(M), f^{-1}(Q) \in \operatorname{Min}(L)$. We remark that $f: L \rightarrow M$ is an $m$-lattice morphism if and only if $f^{\bullet}: \operatorname{Id}(L) \rightarrow \operatorname{Id}(M)$ is an $m$-frame morphism.

If $u: A \rightarrow B$ is an $m$-quantale morphism then we consider the function $\Gamma: \operatorname{Min}(B) \rightarrow \operatorname{Min}(A)$ defined by $\Gamma(q)=\tilde{u}(q)$, for any $q \in \operatorname{Min}(B)$.

Lemma 6.1. Assume that $u$ is an m-quantale morphism.

(1) If $c \in K(A)$, then $\Gamma^{-1}\left(V_{A}(c) \bigcap \operatorname{Min}(A)\right)=V_{B}(u(c)) \bigcap \operatorname{Min}(B)$;

(2) $\Gamma$ is a continuous map w.r.t. the Zariski and the flat topologies.

Let us fix two semiprime coherent quantales $A, B$ and a coherent quantale morphism $f: R \rightarrow S$ According to Proposition 4.2, one can consider the lattice morphism $L(u): L(A) \rightarrow L(B)$.

Lemma 6.2. If $q \in \operatorname{Spec}(B)$, then $(L(u))^{-1}\left(q^{*}\right)=(\tilde{u}(q))^{*}$.

Proof. Assume that $x \in(L(u))^{-1}\left(q^{*}\right)$ so there exists $c \in K(A)$ such that $x=\lambda_{A}(c)$ and $L(u)\left(\lambda_{A}(c)\right) \in q^{*}$. According to Proposition 4.2, we get $u\left(\lambda_{A}(c)\right) \in q^{*}$, hence $u(c) \leq q$ (by Lemma 3.3(8)). By using the adjointness property we get $c \leq \tilde{u}(q)$, hence $x=\lambda_{A}(c) \in(\tilde{u}(q))^{*}$. Thus we obtain the inclusion $(L(u))^{-1}\left(q^{*}\right) \subseteq(\tilde{u}(q))^{*}$. 
In order to prove the converse inclusion $(\tilde{u}(q))^{*} \subseteq(L(u))^{-1}\left(q^{*}\right)$, assume that $d$ is a compact element of $A$ such that $d \leq \tilde{u}(q)$. Then we have $u(d) \leq q$, hence $L(u)\left(\lambda_{A}(d)\right)=\left(\lambda_{B}(u(d)) \in q^{*}\right.$, i.e., $\left.\quad \lambda_{A}(d)\right) \in$ $(L(u))^{-1}\left(q^{*}\right)$. Thus the inclusion $(\tilde{u}(q))^{*} \subseteq(L(u))^{-1}\left(q^{*}\right)$ follows.

Proposition 6.3. The following assertions are equivalent:

(1) $u$ is an m-quantale morphism;

(2) $L(u)$ is an m-lattice morphism.

Proof. $(1) \Rightarrow(2)$ Let $Q$ be a minimal prime ideal of the lattice $L(B)$, so $Q=q^{*}$ for some $q \in \operatorname{Min}(B)$. By the hypothesis that $u$ is an $m$ quantale morphism we have $\tilde{u}(q) \in \operatorname{Min}(A)$, so $(\tilde{u}(q))^{*} \in \operatorname{Min}_{I d}(L(A))$. In accordance with Lemma 6.2, we have $(L(u))^{-1}(Q)=(L(u))^{-1}\left(q^{*}\right)=(\tilde{u}(q))^{*}$, so $(L(u))^{-1}(Q)$ is a minimal prime ideal of the lattice $L(A)$. Then $L(u)$ is an $m$-lattice morphism.

$(2) \Rightarrow(1)$ Assume that $L(u)$ is an $m$-lattice morphism. Let $q$ be a minimal prime element of the quantale $B$, hence $q^{*} \in \operatorname{Min}_{I d}(L(B))$. By taking into account the hypothesis, it follows that $(L(u))^{-1}\left(q^{*}\right) \in$ $\operatorname{Min}_{I d}(L(A))$. By using Lemma 6.2, we get $(\tilde{u}(q))^{*} \in \operatorname{Min}_{I d}(L(A))$, hence $\tilde{u}(q) \in \operatorname{Min}(A)$. We conclude that $u$ is an $m$-quantale morphism. 
Lemma 6.4. The following assertions are equivalent:

(1) $u$ is an m-quantale morphism;

(2) For all $q \in \operatorname{Min}(A)$ and $c \in K(A), c \leq q$ implies $c^{\perp_{A}} \leq \tilde{u}(q)$;

(3) For all $q \in \operatorname{Min}(A)$ and $c \in K(A), c \leq q$ if and only if $c^{\perp_{A}} \leq \tilde{u}(q)$.

Proof. By Proposition 3.10.

Proposition 6.5. If $u: A \rightarrow B$ is an m-quantale morphism, then it is a w-Baer quantale morphism.

Proof. Let $c, d$ be two compact elements of $A$ such that $c^{\perp_{A}}=d^{\perp_{A}}$. Assume by absurdum that $(u(c))^{\perp_{B}} \neq(u(d))^{\perp_{B}}$, so $(u(c))^{\perp_{B}} \npreceq(u(d))^{\perp_{B}}$ or $(u(d))^{\perp_{B}} \leq(u(c))^{\perp_{B}}$. For example, suppose that $(u(c))^{\perp_{B}} \leq(u(d))^{\perp_{B}}$, so there exists $e \in K(B)$ such that $e \leq(u(c))^{\perp} B$ and $e \notin(u(d))^{\perp B}$. Then $e u(d) \neq 0$ so there exists $q \in \operatorname{Min}(B)$ such that $e u(d) \leq q$ (because $\wedge \operatorname{Min}(B)=0)$, hence $e \nless q$ and $u(d) \nless q$. Since $u$ is an $m$-quantale morphism we have $\tilde{u}(q) \in \operatorname{Min}(A)$.

From $e \leq q$ and $e \leq(u(c))^{\perp_{B}}$ we get $u(c) \leq e^{\perp_{B}} \leq q$, hence $c \leq \tilde{u}(q)$ (because $\tilde{u}$ is the right adjoint of $u$ ). Since $\tilde{u}(q) \in \operatorname{Min}(A)$, by applying Proposition 3.10 we get $c^{\perp_{A}} ₫ \tilde{u}(q)$.

From $u(d) \leftleftarrows q$ we get $d \leftleftarrows \tilde{u}(q)$ (by the adjointness property), hence $c^{\perp_{A}}=d^{\perp_{A}} \leq \tilde{u}(q)$. We obtained a contradiction, hence $(u(c))^{\perp_{B}}=(u(d))^{\perp_{B}}$, so $u$ is a $w$-Baer quantale morphism. 
Remark 6.6. According to Proposition 2.4 of [22], if $f: R \rightarrow S$ is a $w$-Baer ring morphism and $\operatorname{Min}_{Z}(R)$ is compact, then $f$ is a minimalisant ring morphism. An open question is if this assertion can be generalised to quantale morphisms: if $u: A \rightarrow B$ is a $w$-Baer quantale morphism and $\operatorname{Min}_{Z}(A)$ is compact, then is $u$ a minimalisant quantale morphism?.

\section{Quasi $r$-Quantale Morphisms}

Let $f: Q \rightarrow S$ be a morphism of commutative rings. We say that $f$ is an $r$-ring morphism (resp., a quasi $r$-ring morphism) if for each $Q \in \operatorname{Min}(S)$ and for each finitely generated ideal $J$ of $S$ such that $J \nsubseteq Q$ there exists an element $a \in R$ (resp., a finitely generated ideal $I$ of $R$ ) such that $f(a) \notin Q$ and $A n n_{S}(s) \subseteq A n n_{S}\left(I^{e}\right)$ (resp., $I^{e} \nsubseteq Q$ and $\left.A n n_{S}(J) \subseteq A n n_{S}\left(I^{e}\right)\right)$.

The notion of quasi $r$-ring morphism can be extended to quantale theory: a quantale morphism $u: A \rightarrow B$ is said to be a quasi $r$-quantale morphism if for all $q \in \operatorname{Min}(B)$ and $d \in K(A)$ such that $d \nless q$ there exists $c \in K(A)$ such that $u(c) \npreceq q$ and $d^{\perp_{B}} \leq(u(c))^{\perp_{B}}$. In a similar way, we define the notion of quasi $r$-frame morphism.

Let us fix two semiprime coherent quantales $A, B$ and a coherent quantale morphism $u: A \rightarrow B$.

Proposition 7.1. Let $u: A \rightarrow B$ be a quasi r-quantale morphism. Then $u$ is an m-quantale morphism and the function $\Gamma: \operatorname{Min}(B) \rightarrow \operatorname{Min}(A)$ is bijective.

Proof. Assume by absurdum that $u: A \rightarrow B$ is not an $m$-quantale morphism, so there exists $q \in \operatorname{Min}(B)$ such that $\tilde{u}(q) \notin \operatorname{Min}(A)$. Then there exists $p \in \operatorname{Min}(A)$ such that $p<\tilde{u}(q)$. According to Corollary 4.5, there exists $r \in \operatorname{Min}(B)$ such that $p=\tilde{u}(r)$, so the minimal prime 
elements $q, r$ of the quantale $B$ are distinct (assuming that $r=q$ we get $\quad p=\tilde{u}(r)=\tilde{u}(q)$, contradicting $\quad p<\tilde{u}(q))$. Thus there exists $d \in K(B)$ such that $d \leq r$ and $d \leftleftarrows q$, hence there exists $c \in K(A)$ such that $u(c) \leq q$ and $d^{\perp_{B}} \leq(u(c))^{\perp_{B}}$ (because $u$ is a quasi $r$-quantale morphism).

By Proposition 3.10, $d \leq r$ and $r \in \operatorname{Min}(A)$ imply $d^{\perp_{B}} \leq r$, hence $(u(c))^{\perp_{B}} \leq r$, therefore $u(c) \leq r$ (because $r$ is an $m$-prime element). By using the adjointness property we have $c \leq \tilde{u}(r)=p<\tilde{u}(q)$, so $u(c)<q$. We obtained a contradiction, so $u$ is an $m$-quantale morphism.

According to Corollary 4.5, for any $p \in \operatorname{Min}(A)$ there exists $q \in \operatorname{Min}(B)$ such that $\tilde{u}(q)=p$, so $\Gamma$ is surjective.

Assume that $q_{1}, q_{2} \in \operatorname{Min}(B)$ and $q_{1} \neq q_{2}$, so there exists $d \in K(B)$ such that $d \leq q_{1}$ and $d \leq q_{2}$. Since $u$ is a quasi $r$-quantale morphism there exists $c \in K(A)$ such that $u(c) \nsubseteq q_{2}$ and $d^{\perp_{B}} \leq(u(c))^{\perp_{B}}$.

In accordance with Corollary 4.5, the following implications hold:

$$
d \leq q_{1} \Rightarrow d^{\perp B} \leftleftarrows q_{1} \Rightarrow(u(c))^{\perp_{B}} \leftleftarrows q_{1} \Rightarrow u(c) \leq q_{1} \Rightarrow c \leq \tilde{u}\left(q_{1}\right) .
$$

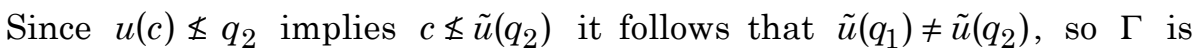
injective.

Theorem 7.2. The following assertions are equivalent:

(1) $u$ is a quasi r-quantale morphism;

(2) The function $\Gamma: \operatorname{Min}_{Z}(B) \rightarrow \operatorname{Min}_{Z}(A)$ is a homeomorphism. 
Proof. $(1) \Rightarrow(2)$ According to Proposition 7.1 , we know that $\Gamma$ is a continuous bijective map. A basic open subset of $\operatorname{Min}_{Z}(B)$ has the form $D_{B}(d)$, where $d$ is a compact element of $B$. We shall prove that $\left.\Gamma\left(D_{B}(d)\right) \bigcap \operatorname{Min}(B)\right)$ is an open subset of $\operatorname{Min}_{Z}(A)$.

We observe that $\Gamma\left(D_{B}(d) \bigcap \operatorname{Min}(B)\right)=\{\tilde{u}(q) \mid q \in \operatorname{Min}(B), d \leftleftarrows q\}$. Let $\tilde{u}(q)$ be a point of $\Gamma\left(D_{B}(d) \bigcap \operatorname{Min}(B)\right)$, i.e., $q \in \operatorname{Min}(B)$ and $d \leftleftarrows q$. Since $u$ is a quasi $r$-quantale morphism, there exists $c \in K(A)$ such that $u(c) \leq q$ and $d^{\perp_{B}} \leq\left((u(c))^{\perp_{A}}\right.$.

We shall show that $\tilde{u}(q) \in D_{A}(c) \bigcap \operatorname{Min}(A) \subseteq \Gamma\left(D_{B}(d) \bigcap \operatorname{Min}(B)\right)$. From $u(c) \leq q$ one obtains $c \leq \tilde{u}(q)$, so $\tilde{u}(q) \in D_{A}(c) \bigcap \operatorname{Min}(A)$. Now let us consider that $p \in D_{A}(c) \bigcap \operatorname{Min}(A)$, so $p \in \operatorname{Min}(A)$ and $c \leftleftarrows p$. By taking into account Corollary 4.5, from $p \in \operatorname{Min}(A)$ it follows that $p=\tilde{u}(r)$, for some $r \in \operatorname{Min}(A)$.

The following implications hold:

$c \leftleftarrows p=\tilde{u}(r) \Rightarrow u(c) \pm r \Rightarrow(u(c))^{\perp_{B}} \leq r \Rightarrow d^{\perp} B \leq r \Rightarrow d \leftleftarrows r \Rightarrow r \in D_{B}(d)$.

Since $p=\tilde{u}(r)=\Gamma(r)$, we obtain the following inclusion:

$$
D_{A}(c) \bigcap \operatorname{Min}(A) \subseteq \Gamma\left(D_{B}(d) \bigcap \operatorname{Min}(B)\right)
$$

$(2) \Rightarrow(1)$ Assume that $\Gamma: \operatorname{Min}_{Z}(B) \rightarrow \operatorname{Min}_{Z}(A)$ is a homeomorphism and consider the elements $q \in \operatorname{Min}(B), d \in K(B)$ such that $d \leftleftarrows q$. Thus $q \in D_{B}(d) \bigcap \operatorname{Min}(B)$, so $\Gamma(q)$ is an element of the open subset $\Gamma\left(D_{B}(d) \bigcap \operatorname{Min}(B)\right)$ of $\operatorname{Min}_{Z}(A)$. Therefore there exists a compact element $c$ of $A$ such that $\Gamma(q) \in D_{A}(c) \bigcap \operatorname{Min}(A) \subseteq \Gamma\left(D_{B}(d) \bigcap \operatorname{Min}(B)\right)$, hence $c \nless \Gamma(q)=\tilde{u}(q)$. By the adjointness property we have $u(c) \npreceq q$. 
In order to prove that $d^{\perp_{B}} \leq(u(c))^{\perp_{B}}$, consider a compact element $e$ of $A$ such that $e \leq d^{\perp_{B}}$, so $e d=0$. For any $r \in \operatorname{Min}(B)$, we have two possibilities:

- If $u(c) \leq r$, then $e u(c) \leq r$;

- If $u(c) \leftleftarrows r$, then $c \leftleftarrows \tilde{u}(r)$, so $\tilde{u}(r) \in D_{A}(c) \bigcap \operatorname{Min}(A)$. It follows that $\Gamma(r)=\tilde{u}(r) \in \Gamma\left(D_{B}(d) \bigcap \operatorname{Min}(B)\right)$, hence $r \in D_{B}(d)$ (because $\Gamma$ is bijective), i.e., $d \leq r$. Thus $e \leq d^{\perp B} \leq r$, so $e u(c) \leq e \leq r$.

Then $e u(c) \leq \wedge \operatorname{Min}(B)=0$, so $e u(c)=0$, i.e., $e \leq(u(c))^{\perp} B$. We conclude that $d^{\perp_{B}} \leq(u(c))^{\perp_{B}}$, hence $u$ is a quasi $r$-quantale morphism.

Let $f: L \rightarrow M$ be a morphism of bounded distributive lattices. Then $f$ is called an $r$-lattice morphism if for all $Q \in \operatorname{Min}_{I d}(M)$ and $y \in M$ such that $y \notin Q$ there exists $x \in L$ such that $f(x) \notin Q$ and $A n n_{M}(y) \subseteq$ $A n n_{M}(f(x))$. We remark that $f$ is an $r$-lattice morphism if and only if $f^{\bullet}: \operatorname{Id}(L) \rightarrow \operatorname{Id}(M)$ is a quasi $r$-frame morphism.

Theorem 7.3. The following assertions are equivalent:

(1) $u: A \rightarrow B$ is a quasi r-quantale morphism;

(2) $L(u): L(A) \rightarrow L(B)$ is an r-lattice morphism.

Proof. $(1) \Rightarrow(2)$ Let us consider an element $y \in L(B)$ and a minimal prime ideal $Q$ of $L(B)$ such that $y \notin Q$. We have to show that there exists $x \in L(A)$ such that $L(u)(x) \notin Q$ and $A n n_{L(B)}(y) \subseteq A n n_{L(B)}(L(u)(x))$. 
Let us take a compact element $d$ of $B$ and a minimal $m$-prime element $q$ of $B$ such that $y=\lambda_{A}(d)$ and $Q=q^{*}$, hence $\lambda_{A}(d) \notin q *$. By Lemma 3.3(8) we have $d \leftleftarrows q$, hence, by applying the hypothesis that $u$ is a quasi $r$-quantale morphism it follows that there exists $c \in K(A)$ such that $u(c) \leq q$ and $d^{\perp B} \leq(u(c))^{\perp_{B}}$.

Denote $x=\lambda_{A}(c)$ and assume by absurdum that $L(u)(x) \in Q$, hence by Proposition 4.2 we have $\lambda_{B}(u(c))=L(u)(u(c))=L(u)(x) \in q^{*}$. By Lemma $3.3(8)$ we get $u(c) \leq q, \quad$ contradicting $\quad u(c) \leq q . \quad$ Then one obtains $L(u)(x) \notin Q$. By applying Proposition 3.8, it results that $A n n_{L(B)}(y)=A n n_{L(B)}\left(\lambda_{B}(d)\right)=A n n_{L(B)}\left(d^{*}\right)=\left(d^{\perp_{B}}\right)^{*}$. Similarly, $A n n_{L(B)}(L(u)(x))=A n n_{L(B)}\left(L(u)\left(\lambda_{A}(c)\right)\right)=A n n_{L(B)}\left(\lambda_{B}(u(c))\right)=A n n_{L(B)}$ $\left((u(c))^{*}\right)=\left((u(c))^{\perp} B\right)^{*}$.

Since $(\cdot)^{*}$ is an order-preserving map, from the inequality $d^{\perp_{B}} \leq$ $(u(c))^{\perp B}$ we get $\left(d^{\perp B}\right)^{*} \subseteq\left((u(c))^{\perp B}\right)^{*}$, so $A n n_{L(B)}(y) \subseteq A n n_{L(B)}(L(u)(x))$. We conclude that $L(u)$ is an $r$-lattice morphism.

(2) $\Rightarrow$ (1) Assume that $q \in \operatorname{Min}(B)$ and $d$ is a compact element of $B$ such that $d \leftleftarrows q$. We have to prove that there exists $c \in K(A)$ such that $u(c) \nsubseteq q$ and $d^{\perp_{B}} \leq(u(c))^{\perp_{B}}$. We observe that $q^{*} \in \operatorname{Min}_{I d}(L(B))$, so $d \nsubseteq q$ implies $\lambda_{B}(d) \notin q^{*}$ (cf. Lemma 3.3(8)). By applying the hypothesis that $L(u): L(A) \rightarrow L(B)$ is an $r$-lattice morphism, there exists $c \in K(A)$ such that $L(u)\left(\lambda_{A}(c)\right) \notin q^{*}$ and $A n n_{L(B)}\left(\lambda_{B}(d)\right) \subseteq A n n_{L(B)}\left(L(u)\left(\lambda_{A}(c)\right)\right)$, hence $\quad \lambda_{B}(u(c))=L(u)\left(\lambda_{A}(c)\right) \notin q^{*}$, hence $\left(d^{\perp B}\right)^{*}=A n n_{L(B)}\left(d^{*}\right)=$ $A n n_{L(B)}\left(\lambda_{B}(d)\right) \subseteq A n n_{L(B)}\left(L(u)\left(\lambda_{A}(c)\right)\right)=A n n_{L(B)}\left(\lambda_{A}(u(c))\right)=A n n_{L(B)}$ $\left((u(c))^{*}\right)=\left((u(c))^{\perp_{B}}\right)^{*}$. 
From $\lambda_{B}(u(c)) \notin q^{*}$ we get $u(c) \nsubseteq q$. By using Lemmas 5.3 and 3.3(7), from the inclusion $\left(d^{\perp_{B}}\right)^{*} \subseteq\left((u(c))^{\perp_{B}}\right)^{*}$ one obtains

$$
d^{\perp_{B}}=\rho\left(d^{\perp_{B}}\right)=\left(\left(d^{\perp_{B}}\right)^{*}\right)_{*} \leq\left(\left((u(c))^{\perp_{B}}\right)^{*}\right)_{*}=\rho\left((u(c))^{\perp_{B}}\right)=(u(c))^{\perp_{B}} .
$$

It follows that $u$ is a quasi $r$-quantale morphism.

\section{Quasi $r^{*}$-Quantale Morphisms}

Let $f: Q \rightarrow S$ be a morphism of commutative rings. We say that $f$ is an $r^{*}$-ring morphism (resp., a quasi $r^{*}$-ring morphism) if for each $Q \in \operatorname{Min}(S)$ and for each finitely generated ideal $J$ of $S$ such that $J \subseteq Q$ there exists an element $a \in R$ (resp., a finitely generated ideal $I$ of $R$ ) such that $f(a) \in Q$ and $A n n_{S}(s) \subseteq A n n_{S}\left(I^{e}\right)$ (resp., $I^{e} \subseteq Q$ and $\left.A n n_{S}(J) \subseteq A n n_{S}\left(I^{e}\right)\right)$.

The notion of quasi $r$-ring morphism can be extended to quantale theory: a quantale morphism $u: A \rightarrow B$ is said to be a quasi $r^{*}$-quantale morphism if for all $q \in \operatorname{Min}(B)$ and $d \in K(A)$ such that $d \leq q$ there exists $c \in K(A)$ such that $u(c) \leq q$ and $(u(c))^{\perp_{B}} \leq d^{\perp_{B}}$.

Let us fix two semiprime coherent quantales $A, B$ and a coherent quantale morphism $u: A \rightarrow B$.

Proposition 8.1. Let $u: A \rightarrow B$ be a quasi $r^{*}$-quantale morphism. Then $u$ is an m-quantale morphism and the function $\Gamma: \operatorname{Min}(B) \rightarrow \operatorname{Min}(A)$ is bijective. 
Proof. In order to prove that $u$ is an $m$-quantale morphism assume that $q \in \operatorname{Min}(B)$, hence $\tilde{u}(q) \in \operatorname{Spec}(A)$ (cf. Lemma 4.1). Then there exists $p \in \operatorname{Min}(A)$ such that $p \leq \tilde{u}(q)$. According to Corollary 4.5, there exists $r \in \operatorname{Min}(B)$ such that $p=\tilde{u}(r)$. Assume by absurdum that the minimal prime elements $q, r$ of the quantale $B$ are distinct, so there exists $d \in K(B)$ such that $d \leq r$ and $d \leftleftarrows q$. Since $u$ be a quasi $r^{*}$-quantale morphism, from $d \leq r$ it follows that there exists $c \in K(A)$ such that $u(c) \leq q \quad$ and $\quad(u(c))^{\perp_{B}} \leq d^{\perp_{B}}$. Thus $c \leq \tilde{u}(r)=p \leq \tilde{u}(q)$, so $\quad u(c) \leq q$, therefore $(u(c))^{\perp_{B}} \nless \leq q$ (cf. Proposition 3.10). On the other hand, $d \leftleftarrows q$ implies $d^{\perp_{B}} \leq q$, so $(u(c))^{\perp_{B}} \leq d^{\perp} B \leq q$. We obtained a contradiction, so $r=q$, therefore $\tilde{u}(q)=\tilde{u}(r)=p \in \operatorname{Min}(A)$. It follows that $u$ is an $m$-quantale morphism.

According to Corollary 4.5, for any $p \in \operatorname{Min}(A)$ there exists $q \in \operatorname{Min}(B)$ such that $\tilde{u}(q)=p$, so $\Gamma$ is surjective. Assume that $q_{1}, q_{2} \in \operatorname{Min}(B)$ and $q_{1} \neq q_{2}$, so there exists $d \in K(B)$ such that $d \leq q_{1}$ and $d \leftleftarrows q_{2}$. Since $u$ is a quasi $r^{*}$-quantale morphism there exists $c \in K(A)$ such that $u(c) \leq q_{1}$ and $(u(c))^{\perp_{B}} \leq d^{\perp_{B}}$. From $d \leftleftarrows q_{2}$ we get $d^{\perp_{B}} \leq q_{2}$, hence $(u(c))^{\perp_{B}} \leq q_{2}$. By applying Proposition 3.10 we get $u(c) \leftleftarrows q_{2}$, hence $q_{1}$ and $q_{2}$ are distinct. Then $\Gamma$ is injective.

Theorem 8.2. The following assertions are equivalent:

(1) $u$ is a quasi $r^{*}$-quantale morphism;

(2) The function $\Gamma: \operatorname{Min}_{F}(B) \rightarrow \operatorname{Min}_{F}(A)$ is a homeomorphism. 
Proof. $\quad(1) \Rightarrow(2)$ We know that $\Gamma: \operatorname{Min}_{F}(B) \rightarrow \operatorname{Min}_{F}(A)$ is a continuous map, and by Proposition 8.1, it is bijective. It remains to show that $\Gamma$ is an open map. A basic open subset of $\operatorname{Min}_{F}(B)$ has the form $V_{B}(d)$, where $d$ is a compact element of $B$. We shall prove that $\Gamma\left(V_{B}(d) \bigcap \operatorname{Min}(B)\right)$ is an open subset of $\operatorname{Min}_{F}(A)$.

Consider a point $\in \Gamma\left(V_{B}(d) \bigcap \operatorname{Min}(B)\right)$, so there exists $q \in V_{B}(d)$ such that $p=\tilde{u}(q)$. Since $u$ is a quasi $r$-quantale morphism, from $d \leq q$ it follows that there exists $c \in K(A)$ such that $u(c) \leq q$ and $((u(c)))^{\perp_{A}}$ $\leq d^{\perp_{B}}$. We shall show that $p \in V_{A}(c) \bigcap \operatorname{Min}(A) \subseteq \Gamma\left(V_{B}(d) \bigcap \operatorname{Min}(B)\right)$. From $c \leq \tilde{u}(q)=p$ one obtains $p \in V_{A}(c) \bigcap \operatorname{Min}(A)$.

Now let us consider a point $r \in V_{A}(c) \bigcap \operatorname{Min}(A)$, so $r \in \operatorname{Min}(A)$ and $c \leq r$. By Corollary 4.5, there exists $s \in \operatorname{Min}(B)$ such that $r=\tilde{u}(s)$, so $c \leq r=\tilde{u}(s)$ implies $u(c) \leq s$. In accordance with Proposition 3.10, we get $(u(c))^{\perp} B \leq s$, hence $d^{\perp_{B}} \npreceq s$, therefore $d \leq s$ (because $s$ is $m$-prime). This implies $s \in V_{B}(d) \bigcap \operatorname{Min}(B)$, hence $r=\Gamma(s) \in \Gamma\left(V_{B}(d) \bigcap \operatorname{Min}(B)\right)$. We conclude that $p \in V_{A}(c) \bigcap \operatorname{Min}(A) \subseteq \Gamma\left(V_{B}(d) \bigcap \operatorname{Min}(B)\right), \quad$ so $\Gamma\left(V_{B}(d) \bigcap \operatorname{Min}(B)\right)$ is an open subset of $\operatorname{Min}_{F}(A)$.

$(2) \Rightarrow(1)$ Assume that $\Gamma: \operatorname{Min}_{F}(B) \rightarrow \operatorname{Min}_{F}(A)$ is a homeomorphism and consider the elements $q \in \operatorname{Min}(B)$ and $d \in K(B)$ such that $d \leq q$. Thus $q \in V_{B}(d) \bigcap \operatorname{Min}(B)$, so $\tilde{u}(q)=\Gamma(q)$ is an element of the open subset $\Gamma\left(V_{B}(d) \bigcap \operatorname{Min}(B)\right)$ of $\operatorname{Min}_{F}(A)$. Thus, there exists a compact element $c$ of $A$ such that $\tilde{u}(q) \in V_{A}(c) \bigcap \operatorname{Min}(A) \subseteq \Gamma\left(V_{B}(d) \bigcap \operatorname{Min}(B)\right)$. From $\tilde{u}(q) \in V_{A}(c)$ we get $c \leq \tilde{u}(q)$, hence $u(c) \leq q$. 
In order to prove that $(u(c))^{\perp_{B}} \leq d^{\perp} B$, consider a compact element $y$ of $A$ such that $y \leq(u(c))^{\perp B}$, so $y u(c)=0$. For any $r \in \operatorname{Min}(B)$, we have two possibilities:

- If $d \leq r$, then $d y \leq r$;

- If $d \pm r$, then $\tilde{u}(r) \pm \Gamma\left(V_{B}(d) \bigcap \operatorname{Min}(B)\right)$ (because $\Gamma$ is a bijection), hence $\tilde{u}(r) \notin V_{B}(c) \bigcap \operatorname{Min}(A)$, i.e., $c \pm \tilde{u}(r)$. Thus $u(c) \nsubseteq r$, so $(u(c))^{\perp_{B}} \leq r$. It follows that $y \leq r$, so $d y \leq r$.

Then we obtain $d y \leq \wedge \operatorname{Min}(B)=0$, so $d y=0$, i.e., $y \leq d^{\perp B}$. We conclude that $(u(c))^{\perp_{B}} \leq d^{\perp_{B}}$, hence $u$ is a quasi $r *$-quantale morphism.

Let $f: L \rightarrow M$ be a morphism of bounded distributive lattices. Then $f$ is called an $r *$-lattice morphism if for all $Q \in \operatorname{Min}_{I d}(M)$ and $y \in Q$ there exists $x \in L$ such that $f(x) \in Q$ and $A n n_{M}(f(x)) \subseteq A n n_{M}(y)$. We remark that $f$ is an $r *$-lattice morphism if and only if $f^{\bullet}: \operatorname{Id}(L) \rightarrow \operatorname{Id}(M)$ is a quasi $r *$-frame morphism.

Theorem 8.3. The following assertions are equivalent:

(1) $u: A \rightarrow B$ is a quasi $r *$-quantale morphism;

(2) $L(u): L(A) \rightarrow L(B)$ is an r*-lattice morphism.

Proof. $(1) \Rightarrow(2)$ Let us consider an element $y \in L(B)$ and a minimal prime ideal $Q$ of $L(B)$ such that $y \in Q$. We have to show that there exists $x \in L(A)$ such that $L(u)(x) \in Q$ and $A n n_{L(B)}(L(u)(x)) \subseteq A n n_{L(B)}(y)$. 
Let us take a compact element $d$ of $B$ and a minimal $m$-prime element $q$ of $B$ such that $y=\lambda_{B}(d)$ and $Q=q^{*}$, hence $\lambda_{B}(d) \in q^{*}$. By Lemma 3.3(8) we have $d \leq q$, hence, by applying the hypothesis that $u$ is a quasi $r *$-quantale morphism it follows that there exists $c \in K(A)$ such that $u(c) \leq q$ and $(u(c))^{\perp_{B}} \leq d^{\perp_{B}}$. From $u(c) \leq q$ we get $L(u)\left(\lambda_{A}(c)\right)=\lambda_{B}(u(c)) \in q^{*}=Q$. If we denote $x=\lambda_{A}(c)$ then it follows that $L(u)(x) \in Q$.

By using the proof of Theorem 7.3, we have $A n n_{L(B)}(y)=\left(d^{\perp B}\right)^{*}$ and $A n n_{L(B)}(L(u)(x))=\left((u(c))^{\perp B}\right)^{*}$. Since $(\cdot)^{*}$ is an order-preserving map, from the inequality $(u(c))^{\perp_{B}} \leq d^{\perp_{B}}$ we get the inclusion $\left((u(c))^{\perp_{B}}\right)^{*}$ $\subseteq\left(d^{\perp_{B}}\right)^{*}$, so $A n n_{L(B)}(L(u)(x)) \subseteq A n n_{L(B)}(y)$. We conclude that $L(u)$ is an $r *$-lattice morphism.

(2) $\Rightarrow(1)$ Assume that $q \in \operatorname{Min}(B)$ and $d$ is a compact element of $B$ such that $d \leq q$. We have to prove that there exists $c \in K(A)$ such that $u(c) \leq q$ and $(u(c))^{\perp_{B}} \leq d^{\perp_{B}}$. We observe that $q^{*} \in \operatorname{Min}_{I d}(L(B))$, so $d \leq q$ implies $\lambda_{B}(d) \in q^{*}$ (cf. Lemma 3.3(8)). By applying the hypothesis that $L(u): L(A) \rightarrow L(B)$ is an $r *$-lattice morphism, there exists $c \in K(A)$ such that $L(u)\left(\lambda_{A}(c)\right) \in q^{*}$ and $A n n_{L(B)}\left(L(u)\left(\lambda_{A}(c)\right)\right) \subseteq$ $A n n_{L(B)}\left(\lambda_{B}(d)\right)$. By using Proposition 4.2 we get $\lambda_{B}(u(c))=\mathrm{\iota}(u)$ $\left(\lambda_{A}(c)\right) \in q^{*}$, therefore $u(c) \leq q$ (by Lemma 3.3(8)).

On the other hand, we have

$$
\begin{aligned}
\left(d^{\perp_{B}}\right)^{*} & =A n n_{L(B)}\left(d^{*}\right)=A n n_{L(B)}\left(\lambda_{B}(d)\right) \supseteq A n n_{L(B)}\left(L(u)\left(\lambda_{A}(c)\right)\right) \\
& =A n n_{L(B)}\left(\lambda_{A}(u(c))\right)=A n n_{L(B)}\left((u(c))^{*}\right)=\left((u(c))^{\perp B}\right)^{*}
\end{aligned}
$$


Recall that $(\cdot)_{*}$ is an order-preserving map. Then by using Lemmas 5.3 and 3.3(7), from the $\left(d^{\perp} B\right)^{*} \supseteq\left((u(c))^{\perp B}\right)^{*}$ one obtains

$$
d^{\perp_{B}}=\rho\left(d^{\perp_{B}}\right)=\left(\left(d^{\perp_{B}}\right)^{*}\right)_{*} \geq\left(\left((u(c))^{\perp_{B}}\right)^{*}\right)_{*}=\rho\left((u(c))^{\perp_{B}}\right)=(u(c))^{\perp_{B}} .
$$

It follows that $u$ is a quasi $r *$-quantale morphism.

\section{Quasi Rigid Quantale Morphisms}

Let $f: R \rightarrow S$ be a morphism of commutative rings. Then $f$ is said to be a quasi rigid ring morphism if for each finitely generated ideal $J$ of $S$ there exists a finitely generated ideal $I$ of $R$ such that $A n n_{S}\left(f^{\bullet}(I)\right)=$ $A n n_{S}(J)$.

Let $A, B$ be two coherent frames. According to $[4,6]$, a frame morphism $u: A \rightarrow B$ is said to be a rigid frame morphism if for each $d \in K(B)$ there exists $c \in K(A)$ such that $(u(c))^{\perp_{B}}=d^{\perp_{B}}$.

Let $A, B$ be two coherent quantales and $u: A \rightarrow B$ a quantale morphism. Then $u$ is said to be a quasi rigid quantale morphism if for each $d \in K(B)$ there exists $c \in K(A)$ such that $(u(c))^{\perp_{B}}=d^{\perp_{B}}$.

If $f: R \rightarrow S$ is a morphism of commutative rings, then $f$ is a quasi rigid ring morphism if and only if $f^{\bullet}: \operatorname{Id}(R) \rightarrow \operatorname{Id}(S)$ is a quasi rigid quantale morphism.

Let us fix two semiprime coherent quantales $A, B$ and a coherent quantale morphism $u: A \rightarrow B$.

Proposition 9.1. If $u: A \rightarrow B$ is a quasi rigid quantale morphism, then it is a quasi r-quantale morphism and a quasi $r *$-quantale morphism. 
Proof. Assume that $q \in \operatorname{Min}(B)$ and $d \in K(B)$. By hypothesis, there exists $c \in K(A)$ such that $(u(c))^{\perp_{B}}=d^{\perp_{B}}$. According to Proposition 3.10, the following implications hold:

(a) $d \leq q \Rightarrow d^{\perp_{B}} \leq q \Rightarrow(u(c))^{\perp_{B}} \leq q \Rightarrow(u(c))^{\perp_{B}} \npreceq q ;$

(b) $d \leq q \Rightarrow d^{\perp_{B}} \leq q \Rightarrow(u(c))^{\perp_{B}} \leq q \Rightarrow(u(c))^{\perp_{B}} \leq q$.

Thus $u$ is a quasi $r$-quantale morphism and a quasi $r *$-quantale morphism.

Corollary 9.2. Assume that $u: A \rightarrow B$ is a quasi rigid quantale morphism. Then $\Gamma: \operatorname{Min}_{Z}(B) \rightarrow \operatorname{Min}_{Z}(A)$ and $\Gamma: \operatorname{Min}_{F}(B) \rightarrow \operatorname{Min}_{F}(A)$ are homeomorphisms.

Proof. We apply Theorems 7.2 and 8.2.

Theorem 9.3. If $u: A \rightarrow B$ is a quasi $r *$-quantale morphism, then the following assertions are equivalent:

(1) $u: A \rightarrow B$ is a quasi rigid quantale morphism;

(2) $\Gamma$ maps basic open sets of $\operatorname{Min}_{F}(B)$ to basic open sets of $\operatorname{Min}_{F}(A)$.

Proof. $\quad(1) \Rightarrow(2)$ A basic open set of $\operatorname{Min}_{F}(B)$ has the form $V_{B}(d) \bigcap \operatorname{Min}(B)$, where $d \in K(B)$. By the hypothesis that $u$ is a quasi rigid quantale morphism we have $(u(c))^{\perp_{B}}=d^{\perp_{B}}$, for some $c \in K(A)$. In order to show that $\Gamma$ maps basic open sets of $\operatorname{Min}_{F}(B)$ to basic open sets of $\operatorname{Min}_{F}(A)$ it suffices to prove that the equality $\Gamma\left(V_{B}(d) \bigcap \operatorname{Min}(B)\right)=$ $V_{A}(c) \bigcap \operatorname{Min}(A)$ holds. 
Assume that $p \in \Gamma\left(V_{B}(d) \bigcap \operatorname{Min}(B)\right)$, hence there exists $q \in \operatorname{Min}(B)$ such that $d \leq q$ and $p=\tilde{u}(q)$. According to Proposition 3.10, the following implications hold: $d \leq q \Rightarrow d^{\perp_{B}} \leq q \Rightarrow(u(c))^{\perp_{B}} \leq q \Rightarrow u(c)$ $\leq q \Rightarrow c \leq \tilde{u}(q) \Rightarrow \tilde{u}(q) \in V_{A}(c) \Rightarrow p=\tilde{u}(q) \in V_{A}(c) \bigcap \operatorname{Min}(A)$. It follows that $\Gamma\left(V_{B}(d) \bigcap \operatorname{Min}(B)\right) \subseteq V_{A}(c) \bigcap \operatorname{Min}(A)$.

In order to prove that $V_{A}(c) \bigcap \operatorname{Min}(A) \subseteq \Gamma\left(V_{B}(d) \bigcap \operatorname{Min}(B)\right)$ we assume that $p \in V_{A}(c) \bigcap \operatorname{Min}(A)$. By using Corollary 4.5, there exists $q \in \operatorname{Min}(B)$ such that $p=\tilde{u}(q)=\Gamma(q)$. By using Proposition 3.10, the following implications hold: $p \in V_{A}(c) \Rightarrow c \leq p=\tilde{u}(q) \Rightarrow u(c) \leq q \Rightarrow(u(c))^{\perp_{B}}$ $\npreceq q \Rightarrow d^{\perp B} \npreceq q \Rightarrow d \leq q \Rightarrow q \in V_{B}(d) \bigcap \operatorname{Min}(B)$. It follows that $p \in \Gamma\left(V_{B}(d)\right.$ $\bigcap \operatorname{Min}(B))$, so we get the desired inclusion.

(2) $\Rightarrow$ (1) Assume that $d \in K(B)$. By using the hypothesis that $\Gamma$ maps basic open sets of $\operatorname{Min}_{F}(B)$ to basic open sets of $\operatorname{Min}_{F}(A)$, there exists $c \in K(A)$ such that $\Gamma\left(V_{B}(d) \bigcap \operatorname{Min}(B)\right)=V_{A}(c) \bigcap \operatorname{Min}(A)$. By Lemma 6.1(1), we have $\Gamma^{-1}\left(V_{A}(c) \bigcap \operatorname{Min}(A)\right)=V_{B}(u(c)) \bigcap \operatorname{Min}(B)$.

According to Proposition 8.1, from the hypothesis that $u$ is a quasi $r *$-quantale morphism it follows that $\Gamma$ is bijective, therefore $V_{B}(d) \bigcap \operatorname{Min}(B)=\Gamma^{-1}\left(V_{A}(c) \bigcap \operatorname{Min}(A)\right)=V_{B}(u(c)) \bigcap \operatorname{Min}(B)$.

Thus for any $q \in \operatorname{Min}(B)$. the following equivalences hold: $d^{\perp_{B}} \leq q$ iff $d \leq q$ iff $u(c) \leq q$ iff $(u(c))^{\perp_{B}} \leq q$ (we used Proposition 3.10). It follows that $V_{B}\left(d^{\perp_{B}}\right) \bigcap \operatorname{Min}(B)=V_{B}\left((u(c))^{\perp_{B}}\right) \bigcap \operatorname{Min}(B)$. By applying Proposition 2.5, we get

$$
d^{\perp_{B}}=\wedge\left(V_{B}\left(d^{\perp_{B}}\right) \bigcap \operatorname{Min}(B)\right)=\Lambda\left(V_{B}\left((u(c))^{\perp_{B}}\right) \bigcap \operatorname{Min}(B)\right)=(u(c))^{\perp_{B}},
$$

therefore $u$ is a quasi rigid quantale morphism. 
Let $f: L \rightarrow M$ be a morphism of bounded distributive lattices. Then $f$ is said to be a rigid lattice morphism if for any $y \in M$ there exists $x \in R$ such that $A n n_{M}(f(x))=A n n_{M}(y)$. It is clear that $f$ is a rigid lattice morphism if and only if $f^{\bullet}: \operatorname{Id}(L) \rightarrow \operatorname{Id}(M)$ is a rigid frame morphism.

Theorem 9.4. The following assertions are equivalent:

(1) $u: A \rightarrow B$ is a quasi rigid quantale morphism;

(2) $L(u): L(A) \rightarrow L(B)$ is a rigid lattice morphism.

Proof. $(1) \Rightarrow(2)$ Assume that $y \in L(B)$ so $y=\lambda_{B}(d)$ for some $d \in K(B)$. According to the hypothesis that $u$ is a quasi rigid quantale morphism there exists $c \in K(A)$ such that $(u(c))^{\perp_{B}}=d^{\perp_{B}}$. By applying Propositions 4.2 and 3.8, the following equalities hold:

$$
\begin{aligned}
A n n_{L(B)}(L(u)(x)) & =A n n_{L(B)}\left(L(u)\left(\lambda_{A}(c)\right)\right)=A n n_{L(B)}\left(\lambda_{B}(u(c))\right) \\
& =A n n_{L(B)}\left(\left((u(c))^{*}\right)=\left((u(c))^{\perp_{B}}\right)^{*}=\left(d^{\perp_{B}}\right)^{*}\right. \\
& =A n n_{L(B)}\left(d^{*}\right)=A n n_{L(B)}\left(\lambda_{B}(d)\right)=A n n_{L(B)}(y) .
\end{aligned}
$$

Thus $L(u)$ is a rigid lattice morphism.

(2) $\Rightarrow(1)$ Let $d$ be a compact element of $B$, so there exists $c \in K(A)$ such that $A n n_{L(B)}\left(L(u)\left(\lambda_{A}(c)\right)=A n n_{L(B)}\left(\lambda_{B}(d)\right)\right.$ (because $L(u)$ is a rigid lattice morphism). By using Proposition 3.8 and Lemma 3.3(5), we get $\left((u(c))^{\perp_{B}}\right)^{*}=A n n_{L(B)}\left(\lambda_{B}(u(c))\right)=A n n_{L(B)}\left(\lambda_{B}(d)\right)=\left(d^{\perp B}\right)^{*}$. 
According to Lemmas 5.3 and 3.3(7), the following equalities hold:

$\left.\left.(u(c))^{\perp_{B}}=\rho\left((u(c))^{\perp_{B}}\right)\right)=\left(\left((u(c))^{\perp_{B}}\right)^{*}\right)_{*}=\left(\left(d^{\perp_{B}}\right)^{*}\right)_{*}=\rho\left((d)^{\perp_{B}}\right)\right)=d^{\perp_{B}}$.

We conclude that $u$ is a quasi rigid quantale morphism.

\section{Acknowledgement}

The author would like to thank the editor and referee for valuable remarks and suggestions which improved the paper.

\section{References}

[1] M. F. Atiyah and I. G. MacDonald, Introduction to Commutative Algebra, AddisonWesley Publishing Company, 1969.

[2] R. Balbes and Ph. Dwinger, Distributive Lattices, University of Missouri Press, 1974.

[3] P. Bhattacharjee, Rigid extensions of algebraic frames, Algebra Universalis 62(1) (2010), 133-149.

DOI: https://doi.org/10.1007/s00012-010-0034-y

[4] P. Bhattacharjee, Minimal Prime Element Space of an Algebraic Frame, Dissertation, Bowling Green State University, 2009.

[5] P. Bhattacharjee, K. M. Dress and W. Wm. McGovern, Extensions of commutative rings, Topology and its Applications 158(14) (2011), 1802-1814.

DOI: https://doi.org/10.1016/j.topol.2011.06.015

[6] P. Bhattacharjee, M. A. Moshier and J. Walters-Wayland, Rigid frame maps and Booleanizations, Topology and its Applications 194 (2015), 400-408.

DOI: https://doi.org/10.1016/j.topol.2015.08.021

[7] G. Birkhoff, Lattice Theory, 3rd Edition, Volume 25, AMS Collocquium Publications, 1967.

[8] D. Cheptea and G. Georgescu, Boolean lifting properties in quantales, Soft Computing 24(8) (2020), 6169-6181.

DOI: https://doi.org/10.1007/s00500-020-04752-8

[9] M. Dickmann, N. Schwartz and M. Tressl, Spectral Spaces, Cambridge University Press, 2019.

DOI: https://doi.org/10.1017/9781316543870 
[10] D. E. Dobbs, M. Fontana and I. Papick, On the flat spectral topology, Rendiconti di Matematica 1(4) (1981), 559-578.

[11] P. Eklund, J. G. Garcia, U. Hohle and J. Kortelainen, Semigroups in Complete Lattices: Quantales, Modules and Related Topics, Springer, 2018.

DOI: https://doi.org/10.1007/978-3-319-78948-4

[12] N. Galatos, P. Jipsen, T. Kowalski and H. Ono, Residuated Lattices: An Algebraic Glimpse at Structural Logics, Studies in Logic and the Foundation of Mathematics, 151, Elsevier, 2007.

[13] G. Georgescu, The reticulation of a quantale, Revue Roumaine des Mathematiques Pures et Appliquees 40(7) (1995), 619-631.

[14] G. Georgescu, Flat topology on the spectra of quantales, Fuzzy Sets and Systems 406 (2021), 22-41.

DOI: https://doi.org/10.1016/j.fss.2020.08.009

[15] M. Hochster, Prime ideals structure in commutative rings, Transactions of the American Mathematical Society 142 (1969), 43-60.

DOI: https://doi.org/10.1090/S0002-9947-1969-0251026-X

[16] P. Jipsen, Generalizations of Boolean products for lattice-ordered algebras, Annals of Pure and Applied Logic 161(2) (2009), 228-234.

DOI: https://doi.org/10.1016/j.apal.2009.05.005

[17] P. T. Johnstone, Stone Spaces, Cambridge University Press, 1982.

[18] K. Keimel, A unified theory of minimal prime ideals, Acta Mathematica Academiae Scientiarum Hungarica 23(2) (1972), 51-69.

DOI: https://doi.org/10.1007/BF01889903

[19] J. Martinez, Abstract Ideal Theory: Ordered Algebraic Structures, Lecture Notes in Pure and Applied Mathematics, 99, Marcel Dekker, New York, 1985, pp. 125-138.

[20] E. Matlis, The minimal prime spectrum of a reduced ring, Illinois Journal of Mathematics 27(3) (1983), 353-391.

DOI: https://doi.org/10.1215/ijm/1256046365

[21] J. Paseka and J. Rosicky, Quantales, Current Research in Operational Quantum Logic: Algebras, Categories and Languages, Fundamental Theories of Physics, Vol. 111, Kluwer, 2000, 245-261.

[22] G. Picavet, Ultrafiltres sur une espace spectral: Anneaux de Baer - Anneaux a spectre maximal compacts, Mathematica Scandinavica 46(1) (1980), 23-53.

[23] K. I. Rosenthal, Quantales and their Applications, Longman Scientific and Technical, 1989. 
[24] H. Simmons, Reticulated rings, Journal of Algebra 66(1) (1980), 169-192.

DOI: https://doi.org/10.1016/0021-8693(80)90118-0

[25] H. Simmons, An introduction of Idioms, 2014.

http:www.cs.man.ac.uk/ hsimmons/00-IDSandMODS/001I2

[26] T. P. Speed, Spaces of ideals of distributive lattices II: Minimal prime ideals, Journal of the Australian Mathematical Society 18(1) (1974), 54-72.

DOI: https://doi.org/10.1017/S144678870001911X

[27] S. H. Sun, Spectra of monoidal-lattices, Algebra Universalis 31(2) (1994), 274-292.

DOI: https://doi.org/10.1007/BF01236523

[28] A. Tarizadeh, Flat topology and its dual aspects, Communications in Algebra 47(1) (2019), 195-205.

DOI: https://doi.org/10.1080/00927872.2018.1469637

[29] A. Tarizadeh, Zariski compactness of minimal spectrum and flat compactness of maximal spectrum, Journal of Algebra and its Applications 18(11) (2019); Article 1950202 .

DOI: https://doi.org/10.1142/S0219498819502025 


\section{Appendix}

Let $R \subseteq S$ be an extension of commutative rings. In ([5], p. 1805) were defined the following types of ring extensions:

- $R \subseteq S$ is a rigid (resp., a quasi rigid) extension if for any $y \in S$ there exists an element $x \in R$ (resp., a finitely generated ideal $I$ of $R$ ) such $A n n_{S}(y)=A n n_{S}(x)\left(\right.$ resp., $\left.A n n_{S}(y)=A n n_{S}\left(I^{e}\right)\right) ;$

- $R \subseteq S$ is an $r$-extension (resp., a quasi $r$-extension) if for all $Q \in \operatorname{Min}(S)$ and $y \in S-Q$ there exists an element $x \in R$ (resp., a finitely generated ideal $I$ of $R$ ) such that $x \notin Q$ and $A n n_{S}(y) \subseteq A n n_{S}(x)$ (resp., $I^{e} \nsubseteq Q$ and $A n n_{S}(y) \subseteq A n n_{S}\left(I^{e}\right)$ );

- $R \subseteq S$ is an $r *$-extension (resp., a quasi $r *$-extension) if for all $Q \in \operatorname{Min}(S)$ and $y \in S-Q$ there exists an element $x \in R$ (resp., a finitely generated ideal $I$ of $R$ ) such that $x \in Q$ and $A n n_{S}(y) \supseteq A n n_{S}(x)$ (resp., $I^{e} \subseteq Q$ and $A n n_{S}(y) \supseteq A n n_{S}\left(I^{e}\right)$ ).

Remark 10.1. For any ring extension $R \subseteq S$, the following equivalences hold:

- $R \subseteq S$ is quasi rigid iff for any finitely generated ideal $J$ of $S$ there exists a finitely generated ideal $I$ of $R$ such that $A n n_{S}(J)=A n n_{S}\left(I^{e}\right)$;

- $R \subseteq S$ is a quasi $r$-extension iff for each $Q \in \operatorname{Min}(S)$ and for each finitely generated ideal $J$ of $S$ such that $J \nsubseteq Q$ there exists a finitely generated ideal $I$ of $R$ such that $I^{e} \nsubseteq Q$ and $A n n_{S}(J) \subseteq A n n_{S}\left(I^{e}\right)$;

- $R \subseteq S$ is a quasi $r *$-ring extension iff for each $Q \in \operatorname{Min}(S)$ and for each finitely generated ideal $J$ of $S$ such that $J \subseteq Q$ there exists a finitely generated ideal $I$ of $R$ such that $I^{e} \subseteq Q$ and $A n n_{S}(J) \supseteq A n n_{S}\left(I^{e}\right)$. 
The previous remark allows us to define the corresponding types of ring morphisms, then to formulate the definition of quasi rigid, quasi $r$-and quasi $r *$-quantale morphisms (see Sections 7-9). We observe that the notions of rigid, $r$ - and $r *$-quantale morphisms cannot be defined in the framework of quantales. 